\title{
SMT-Based Observer Design for Cyber-Physical Systems under Sensor Attacks
}

\author{
YASSER SHOUKRY, UC Berkeley and UC Los Angeles, USA \\ MICHELLE CHONG, Lund University, Sweden \\ MASASHI WAKAIKI, Kobe University, Japan \\ PIERLUIGI NUZZO, University of Southern California, USA \\ ALBERTO SANGIOVANNI-VINCENTELLI and SANJIT A. SESHIA, University of California \\ at Berkeley, USA \\ JOÃO P. HESPANHA, University of California at Santa Barbara, USA \\ PAULO TABUADA, University of California at Los Angeles, USA
}

\begin{abstract}
We introduce a scalable observer architecture, which can efficiently estimate the states of a discrete-time linear-time-invariant system whose sensors are manipulated by an attacker, and is robust to measurement noise. Given an upper bound on the number of attacked sensors, we build on previous results on necessary and sufficient conditions for state estimation, and propose a novel Multi-Modal Luenberger (MML) observer based on efficient Satisfiability Modulo Theory (SMT) solving. We present two techniques to reduce the complexity of the estimation problem. As a first strategy, instead of a bank of distinct observers, we use a family of filters sharing a single dynamical equation for the states, but different output equations, to generate estimates corresponding to different subsets of sensors. Such an architecture can reduce the memory usage of the observer from an exponential to a linear function of the number of sensors. We then develop an efficient SMT-based decision procedure that is able to reason about the estimates of the MML observer to detect at runtime which sets of sensors are attack-free, and use them to obtain a correct state estimate. Finally, we discuss two optimization-based algorithms that can efficiently select the observer parameters with the goal of minimizing the sensitivity of the estimates with respect to sensor noise. We provide proofs of convergence for our estimation algorithm and report simulation results to compare its runtime performance with alternative techniques. We show that our algorithm scales well for large systems (including up to 5,000 sensors) for which many previously proposed algorithms are not implementable due to excessive memory and time requirements. Finally, we illustrate the effectiveness of our approach, both in terms of resiliency to attacks and robustness to noise, on the design of large-scale power distribution networks.
\end{abstract}

This work was partially sponsored by DARPA under agreement number FA8750-12-2-0247, by TerraSwarm, one of six centers of STARnet, a Semiconductor Research Corporation program sponsored by MARCO and DARPA, by the NSF project ExCAPE: Expeditions in Computer Augmented Program Engineering (awards 1138996 and 1139138), and NSF awards CNS1136174, CNS-1329650, and ECCS-1608880.

Authors' addresses: Y. Shoukry, A. Sangiovanni-Vincentelli, and S. A. Seshia, Department of Electrical Engineering and Computer Sciences, University of California, Cory Hall, Berkeley, CA 94720, USA; email: \{yshoukry, alberto, sseshia\}@eecs. berkeley.edu; M. Chong, Department of Automatic Control, Lund University, Ole Römers väg 1, 22363 Lund, Sweden; email: michelle.chong@control.lth.se; M. Wakaiki, Graduate School of System Informatics, Kobe University, 1-1 Rokkodai, Nada, Kobe, 657-8501, Japan; email: wakaiki@ruby.kobe-u.ac.jp; P. Nuzzo, Department of Electrical Engineering, University of Southern California, 3740 McClintock Ave, Los Angeles, CA 90089, USA; email: nuzzo@usc.edu; J. P. Hespanha, Department of Electrical and Computer Engineering, Harold Frank Hall, University of California, Santa Barbara, CA 93106-9560 USA; email: hespanha@ece.ucsb.edu; P. Tabuada, Department of Electrical Engineering, University of California, 56-125B Engineering IV Building, 420 Westwood Plaza (Box 951594), Los Angeles, CA 90095-1594, USA; email: tabuada@ee.ucla.edu. Permission to make digital or hard copies of all or part of this work for personal or classroom use is granted without fee provided that copies are not made or distributed for profit or commercial advantage and that copies bear this notice and the full citation on the first page. Copyrights for components of this work owned by others than the author(s) must be honored. Abstracting with credit is permitted. To copy otherwise, or republish, to post on servers or to redistribute to lists, requires prior specific permission and/or a fee. Request permissions from permissions@acm.org.

(c) 2018 ACM 2378-962X/2018/01-ART5 $\$ 15.00$

https://doi.org/10.1145/3078621

ACM Transactions on Cyber-Physical Systems, Vol. 2, No. 1, Article 5. Publication date: January 2018. 
Categories and Subject Descriptors: K.6.5 [Management of Computing and Information Systems]: Security and Protection-Unauthorized access; D.2.8 [Special-purpose and Application-based Systems]: Process control systems

General Terms: Theory

Additional Key Words and Phrases: Secure state estimation, satisfiability modulo theory, secure cyberphysical systems

\section{ACM Reference format:}

Yasser Shoukry, Michelle Chong, Masashi Wakaiki, Pierluigi Nuzzo, Alberto Sangiovanni-Vincentelli, Sanjit A. Seshia, João P. Hespanha, and Paulo Tabuada. 2018. SMT-Based Observer Design for Cyber-Physical Systems Under Sensor Attacks. ACM Trans. Cyber-Phys. Syst. 2, 1, Article 5 (January 2018), 27 pages.

https://doi.org/10.1145/3078621

\section{INTRODUCTION}

Cyber-physical systems (CPSs) offer a promising response to key infrastructural and societal problems, from efficient transportation to large-scale energy distribution, from security to affordable health-care. In these systems, e.g., power grids, water and gas distribution networks, semiautonomous cars, and wearable devices, sensors and cyber components (e.g., digital processors and networks) tightly interact with physical processes to enhance their capabilities. However, cyber components are also the vehicle of unprecedented vulnerabilities to malicious attacks. Remarkable examples of adversarial attacks include the Stuxnet virus targeting SCADA systems (Langner 2011) as well as the injection of false data in power systems (Liu et al. 2009) and water control systems (Smith 2011), or the non-invasive sensor spoofing attacks in automotive systems (Shoukry et al. 2013). Independently of the nature of the attack, i.e., whether it originates from the cyber or physical portion of the system, it eventually results in corrupted sensor measurements. Using these measurements to control the operation of safety-critical CPSs can finally lead to life-threatening situations.

A viable strategy to achieve resiliency to adversarial attacks in CPSs is to equip them with algorithms that are able to recognize the presence of a sensor attack and reconstruct the actual system state independently of it, by relying on the measurements collected from uncorrupted sensors. This is the approach advocated in this article.

The problem of state reconstruction in the presence of attacks, also denoted as secure state estimation, has recently attracted considerable attention from the control community (Fawzi et al. 2014; Pasqualetti et al. 2013; Chong et al. 2014; Pajic et al. 2014; Shoukry and Tabuada 2016; Mo and Murray 2015; Shoukry et al. 2014; Mishra et al. 2015; Teixeira et al. 2015; Yong et al. 2015). A first category of works (Fawzi et al. 2014; Pajic et al. 2014; Shoukry et al. 2014; Shoukry and Tabuada 2016; Mo and Murray 2015; Teixeira et al. 2015) performs state estimation by analyzing the sensor information collected within a time window of finite length. This is, for instance, the case for the algorithms based on solving an $L_{0}$ optimization problem, proposed by Fawzi et al. (2014) and Pajic et al. (2014), the game-theoretic approach proposed by Mo and Murray (2015), the Gramian-based observer by Chong et al. (2014), and the simultaneous input and state estimation method by Yong et al. (2015). A second category of results in the literature focuses, instead, on designing recursive observers and filters (Shoukry and Tabuada 2016; Chong et al. 2014; Pasqualetti et al. 2013; Mishra et al. 2015). Observers and filters show higher promise of scalability, since they are able to incorporate new information as it becomes available in real time. Moreover, they offer better performance in the presence of noise and modeling errors, since they make use of all the available measurements from the beginning of the observation window to the current time instant $t$. Unfortunately, 
however, because of the combinatorial nature of the secure state estimation problem, the solutions proposed in the literature (Chong et al. 2014; Pasqualetti et al. 2013; Mishra et al. 2015; Yong et al. 2015) usually require a large bank of observers running in parallel, and exhaustive search over the outputs of the different observers, to identify the attacks. Observer-based designs would then be ineffective for large-scale systems unless they are coupled with an efficient procedure that can quickly identify and disregard the malicious measurements.

The problem of reconstructing the state under sensor attacks is also closely related to faulttolerant state reconstruction. Specifically, the robust Kalman filter described by Mattingley and Boyd (2010) and robust Principal Component Analysis (PCA) (Farahmand et al. 2011) are connected with the results in this article. In robust Kalman filtering the state estimate updates are obtained by solving a convex $L_{1}$ optimization problem that is robust to outliers. Similarly, a PCA robust to outliers is developed by Farahmand et al. (2011). However, no theoretical guarantees are known regarding the performance of these techniques in the presence of malicious attacks. Another related area is the design of resilient control algorithms that can operate under the worst case attack scenarios. Such design can be carried over using $H_{\infty}$ and game theoretic techniques (Zhu and Başar 2011).

The complexity of CPSs is arguably the hardest challenge for the deployment of resilient and secure designs. Identifying which sensors are under attack is combinatorial in the number of sensors. While brute-force strategies show poor scalability (Mishra et al. 2015; Chong et al. 2014; Pasqualetti et al. 2013), problem relaxations tend to weaken the security guarantees (Shoukry and Tabuada 2016). The situation is exacerbated by the "trillion devices" scenario posed by the Internet of Things. Devising scalable algorithmic solutions to secure CPS design is, therefore, highly desirable (Nuzzo et al. 2015).

In this article, we address the design of observers that can accurately reconstruct the state of a cyber-physical system under sensor attacks, while being suitable to be deployed in real time and on large-scale systems. At the heart of our approach is a Satisfiability Modulo Theory (SMT) based algorithm which further improves on the scalability and robustness of our previous solutions (Shoukry et al. 2014; Shoukry et al. 2015) while addressing both memory and runtime efficiency. In particular, in our previous work (Shoukry et al. 2014; Shoukry et al. 2015), the system state was estimated from data collected within an observation window of finite length. However, when bounded noise exists in the system, estimating the state using a finite amount of data leads to poor performance in terms of the estimation error (Shoukry et al. 2014). In this article, we propose, instead, to design a Luenberger-like observer that can incorporate new measurements as they become available. Thanks to the recursive nature of the observer, noise is "averaged out" as new information becomes available. Our overall contributions can be summarized as follows:

-We propose, to the best of our knowledge, the first state estimation algorithm that combines the robustness of an observer to bounded noise with the efficiency of SMT-based detection of corrupted sensors.

- We present a novel observer architecture whose memory usage scales linearly with the number of system states and sensors.

- We propose two efficient algorithms to select the observer parameters with the goal of minimizing the sensitivity of the estimates with respect to sensor noise.

- We demonstrate the scalability and effectiveness of our approach on the problem of detecting attacks in large-scale dynamical systems, including large power distribution networks, showing that our observer outperforms previously proposed techniques and is robust to measurement noise. 
In a preliminary version of the results in this article (Shoukry et al. 2016), we mostly focused on the ideal scenario of a system not affected by sensor noise and model inaccuracies. In this article, we provide additional details on the proofs of the theorems introduced in our previous submission. Moreover, we consolidate our results by discussing how the observer parameters can be selected with the goal of maximizing robustness against sensor noise, and by illustrating two optimizationbased algorithms that efficiently provide a sub-optimal choice of parameters via convex relaxations in an automatic way.

The rest of the article is organized as follows. Section 2 presents the mathematical formulation of our problem while Section 3 introduces the overall observer architecture. The design is detailed in Sections 4 and 5. In Section 6, we provide the theoretical analysis of the estimation algorithm and its convergence guarantees in the presence of noise and modeling imperfections. In Section 7, we discuss optimization-based algorithms for robust observer design. Numerical experiments showing scalability and robustness of the proposed approach for large-scale dynamical systems, including power distribution network examples, are offered in Sections 8 and 9. Finally, Section 10 concludes the article.

\section{PROBLEM FORMULATION}

\subsection{Preliminaries}

The symbols $\mathbb{N}, \mathbb{R}, \mathbb{R}^{+}$, and $\mathbb{B}$ denote the sets of natural, real, non-negative real, and Boolean numbers, respectively. The symbols $\wedge$ and $\neg$ denote the logical AND and logical NOT operators, respectively. The support of a vector $x \in \mathbb{R}^{n}$, denoted by $\operatorname{supp}(x)$, is the set of indices of the nonzero elements of $x$. If $S$ is a set, $|S|$ is the cardinality of $S$. For a vector $x \in \mathbb{R}^{n}$, we denote by $\|x\|_{2}$ the 2-norm of $x$ and by $\|M\|_{2}$ the induced 2-norm of a matrix $M \in \mathbb{R}^{m \times n}$. We also denote by $M_{i} \in$ $\mathbb{R}^{1 \times n}$ the $i$ th row of $M$. Similarly, for the set $\Gamma \subseteq\{1, \ldots, m\}$, we denote by $M_{\Gamma} \in \mathbb{R}^{|\Gamma| \times n}$ the matrix obtained from $M$ by removing all the rows except those indexed by $\Gamma$. We use $\lambda_{1}\{M\}, \ldots, \lambda_{n}\{M\}$ to denote the eigenvalues of a matrix $M \in \mathbb{R}^{n \times n}$. A symmetric matrix $M$ is positive definite, written $M>0$, if and only if all its eigenvalues are positive. Finally, for two symmetric matrices $M$ and $N$, we use the notation $M>N$ to express that the matrix $M-N$ is positive definite, i.e., $M-N>0$.

\subsection{System and Attack Model}

We consider a system under sensor attacks of the form

$$
\begin{aligned}
x^{(t+1)} & =A x^{(t)}+B u^{(t)}+\mu^{(t)}, \\
y_{i}^{(t)} & = \begin{cases}C_{i} x^{(t)}+\psi_{i}^{(t)} & \text { if } i \text { th sensor is attack-free } \\
C_{i} x^{(t)}+a_{i}^{(t)}+\psi_{i}^{(t)} & \text { if } i \text { th sensor is under attack, }\end{cases}
\end{aligned}
$$

where $x^{(t)} \in \mathbb{R}^{n}$ is the system state at time $t \in \mathbb{N}, u^{(t)} \in \mathbb{R}^{m}$ is the system input, and $y_{i}^{(t)} \in \mathbb{R}$ is the observed output from the $i$ th sensor where $i \in\{1, \ldots, p\}$. Matrices $A, B$, and $C_{1}, \ldots, C_{p}$ represent the system dynamics and have appropriate dimensions. An attacker can corrupt the sensor measurements $y_{i}$ by either spoofing the sensor output or manipulating the data transmitted from the sensor to the controller. Independently of the nature of the attack, its effect can be described by the attack signal $a_{i}^{(t)}$. We do not assume bounds, statistical properties, or restrictions on the time evolution of the elements in $a_{i}^{(t)}$. We only assume that the attacker has access to a fixed subset of sensors of cardinality $s \leq \bar{s}$; whether a specific sensor in this subset is attacked or not may change with time. As shown by Shoukry and Tabuada (2016, Theorem 3.2), Fawzi et al. (2014, 
Proposition 2), and (Chong et al. 2014, Theorem 1), ${ }^{1}$ the upper bound on the maximum number of sensors under attack $\bar{s}$ is a characteristic of the system and can be computed $a$ priori from the system parameters (the number of sensors $p$ and the $A$ and $C_{i}$ matrices). We will elaborate on how this upper bound is exploited in the design of an observer in Section 3. Finally, the vectors $\mu^{(t)} \in \mathbb{R}^{n}$ and $\psi^{(t)}=\left(\psi_{1}^{(t)}, \ldots, \psi_{p}^{(t)}\right) \in \mathbb{R}^{p}$ represent, respectively, the process (i.e., modeling) noise and the measurement noise, which we assume to be bounded, i.e., there exist bounds $\bar{\psi}_{i}$ and $\bar{\mu}$ such that

$$
\left\|\psi_{i}^{(t)}\right\|_{2} \leq \bar{\psi}_{i}, \quad\left\|\mu^{(t)}\right\|_{2} \leq \bar{\mu}, \quad \forall t \in \mathbb{N}, \forall i \in\{1, \ldots, p\} .
$$

\subsection{Observer Design Problem}

We assume that each sensor can be in one of two modes, i.e., either attack-free or under attack. The attacker has access to at most $\bar{s}$ sensors and the attacker can, at any point of time, decide to switch his attack signal on or off. Since at most $\bar{s}$ sensors can be corrupted, the system can be, at each time, in any of $\sum_{k=0}^{\bar{s}}\left(\begin{array}{c}p \\ p-k\end{array}\right)$ modes, corresponding to the specific sets of sensors being under attack.

Since the attacked sensors are unknown a priori, designing a secure Luenberger observer entails two main steps. We first need to detect the system mode by identifying the sensors which are under attack. Then, we can construct the state estimate from the attack-free sensors. We formally define our task as follows.

Problem 2.1 (Secure Luenberger Observer Design). Given the linear system under attack defined in Equations (2.1) and (2.2), construct an estimate $\hat{x}^{(t)}$ such that

$$
\limsup _{t \rightarrow \infty}\left\|x^{(t)}-\hat{x}^{(t)}\right\|_{2} \leq \rho(\bar{\psi}, \bar{\mu})
$$

for some constant $\rho \in \mathbb{R}^{+}$which depends on the noise bounds $\bar{\psi}=\left(\sum_{i=1}^{p} \bar{\psi}_{i}^{2}\right)^{\frac{1}{2}}$ and $\bar{\mu}$ and such that $\rho=0$ whenever $\bar{\psi}=\bar{\mu}=0$.

In other words, we are interested in an estimate $\hat{x}^{(t)}$ such that the norm of the state estimation error $\left\|x^{(t)}-\hat{x}^{(t)}\right\|_{2}$ converges to a ball centered at the origin and whose radius is just a function of the noise bound. In particular, in the noiseless case (i.e., when $\bar{\psi}=\bar{\mu}=0$ ), $\rho$ is equal to zero and the observer asymptotically converges to the actual system state, independently of the attack.

We observe that estimating the state of a system in the presence of sensor attacks is not always feasible, in general. To establish conditions under which such an estimation is indeed feasible, hence the secure Luenberger observer design problem can be solved, we resort to the notion of s-sparse observability for discrete-time systems (Shoukry and Tabuada 2016) (or, similarly, that of $M$-attack observability (Chong et al. 2014)) defined as follows.

Definition 2.2 (s-Sparse Observable System). The linear control system under attack defined by Equations (2.1) and (2.2) is said to be $s$-sparse observable if for every set $\Gamma \subseteq\{1, \ldots, p\}$ with $|\Gamma|=p-s$, the pair $\left(A, C_{\Gamma}\right)$ is observable.

Informally, a system is $s$-sparse observable if it remains observable after eliminating any choice of $s$ sensors. In the absence of sensor and process noise, the conditions under which the state can be estimated in spite of sensor attacks were studied by Fawzi et al. (2014), Shoukry and Tabuada (2016), and Chong et al. (2014), where it is shown that, if $\bar{s}$ is the maximum number of corrupted sensors, $2 \bar{s}$-sparse observability is necessary and sufficient for secure state estimation. Therefore,

\footnotetext{
${ }^{1}$ This result was derived for continuous-time linear-time-invariant systems.
} 
in what follows, we assume that the $2 \bar{s}$-sparse observability condition always holds. Moreover, we introduce below a convenient and compact notation to describe the main results of this article.

\subsection{Notation}

For a set of $\tau \in \mathbb{N}$ measurements (with $\tau \leq n$ ), we can arrange the outputs from the $i$ th sensor at different time instants as follows:

$$
\widetilde{Y}_{i}^{(t)}=O_{i} x^{(t)}+E_{i}^{(t)}+F_{i} U^{(t)}+\Psi_{i}^{(t)},
$$

where

$$
\begin{aligned}
& \widetilde{Y}_{i}^{(t)}=\left[\begin{array}{c}
y_{i}^{(t)} \\
y_{i}^{(t+1)} \\
\vdots \\
y_{i}^{(t+\tau-1)}
\end{array}\right], E_{i}^{(t)}=\left[\begin{array}{c}
a_{i}^{(t)} \\
a_{i}^{(t+1)} \\
\vdots \\
a_{i}^{(t+\tau-1)}
\end{array}\right], U^{(t)}=\left[\begin{array}{c}
u^{(t)} \\
u^{(t+1)} \\
\vdots \\
u^{(t+\tau-1)}
\end{array}\right], F_{i}=\left[\begin{array}{ccccc}
0 & 0 & \ldots & 0 & 0 \\
C_{i} B & 0 & \ldots & 0 & 0 \\
\vdots & & \ddots & \vdots \\
C_{i} A^{\tau-2} B & C_{i} A^{\tau-3} B & \ldots & C_{i} B & 0
\end{array}\right], \\
& \Psi_{i}^{(t)}=\left[\begin{array}{ccccc}
0 & 0 & \ldots & 0 & 0 \\
C_{i} & 0 & \ldots & 0 & 0 \\
\vdots & & \ddots & \vdots \\
C_{i} A^{\tau-2} & C_{i} A^{\tau-3} & \ldots & C_{i} & 0
\end{array}\right]\left[\begin{array}{c}
\mu^{(t)} \\
\mu^{(t+1)} \\
\vdots \\
\mu^{(t+\tau-1)}
\end{array}\right]+\left[\begin{array}{c}
\psi_{i}^{(t)} \\
\psi_{i}^{(t+1)} \\
\vdots \\
\psi_{i}^{(t+\tau-1)}
\end{array}\right], O_{i}=\left[\begin{array}{c}
C_{i} \\
C_{i} A \\
\vdots \\
C_{i} A^{\tau-1}
\end{array}\right] .
\end{aligned}
$$

Since all the inputs in $U^{(t)}$ are known, we can further simplify the output equation as

$$
Y_{i}^{(t)}=O_{i} x^{(t)}+E_{i}^{(t)}+\Psi_{i}^{(t)},
$$

where $Y_{i}^{(t)}=\widetilde{Y}_{i}^{(t)}-F_{i} U^{(t)}$. We also define

$$
Y^{(t)}=\left[\begin{array}{c}
Y_{1}^{(t)} \\
\vdots \\
Y_{p}^{(t)}
\end{array}\right], E^{(t)}=\left[\begin{array}{c}
E_{1}^{(t)} \\
\vdots \\
E_{p}^{(t)}
\end{array}\right], \Psi^{(t)}=\left[\begin{array}{c}
\Psi_{1}^{(t)} \\
\vdots \\
\Psi_{p}^{(t)}
\end{array}\right], O=\left[\begin{array}{c}
O_{1} \\
\vdots \\
O_{p}
\end{array}\right]
$$

to denote, respectively, the vector of outputs, attacks, noise, and observability matrices related to all sensors over the same time window of length $\tau$. With some abuse of notation, $Y_{i}, E_{i}$, and $O_{i}$ are also used to denote the $i$ th block of $Y, E$, and $O$. Similarly, we denote with $Y_{\Gamma}, E_{\Gamma}, \Psi_{\Gamma}$, and $O_{\Gamma}$ the blocks indexed by the elements in the set $\Gamma$.

Because of our assumptions on the system noise, there exists a uniform upper bound on its magnitude, denoted by $\bar{\Psi}_{i} \in \mathbb{R}^{+}$, i.e., the following inequality $\left\|\Psi_{i}^{(t)}\right\|_{2} \leq \bar{\Psi}_{i}$ holds for all time $t \in \mathbb{N}$. With some abuse of notation, for the set $\Gamma \subseteq\{1, \ldots, p\}$ we denote with $\bar{\Psi}_{\Gamma} \in \mathbb{R}^{+}$the bound on the noise for the set of sensors indexed by $\Gamma$, i.e.,

$$
\left\|\Psi_{\Gamma}^{(t)}\right\|_{2}^{2}=\sum_{i \in \Gamma}\left\|\Psi_{i}^{(t)}\right\|_{2}^{2} \leq \sum_{i \in \Gamma} \bar{\Psi}_{i}^{2}=\bar{\Psi}_{\Gamma}^{2} .
$$

By the same abuse of notation, we drop the subscript $\Gamma$ for the special case in which $\Gamma$ is the set of all sensors, i.e., $\bar{\Psi}=\bar{\Psi}_{\Gamma}$ when $\Gamma=\{1, \ldots, p\}$.

\section{OBSERVER ARCHITECTURE}

In this section, we introduce the overall architecture of the proposed observer. The details of the search algorithms employed in the design are provided in the next section. 


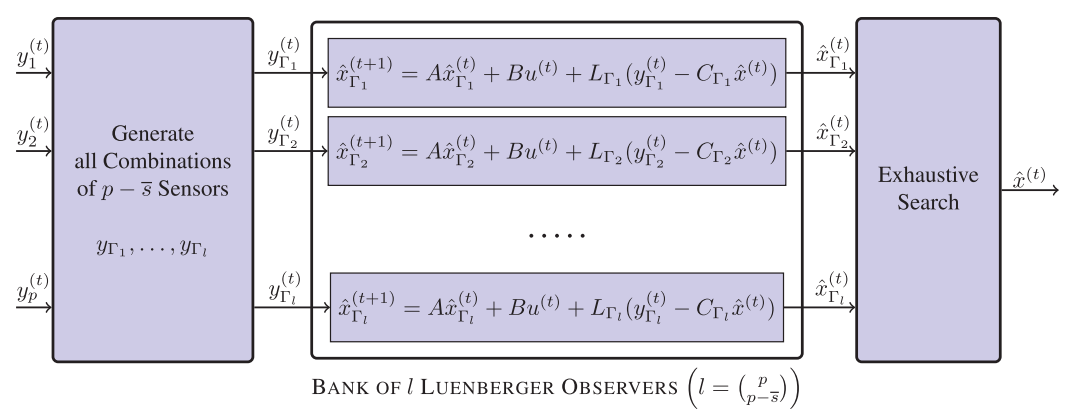

Fig. 1. Architecture of the exhaustive search-based observer. The observer consists of $\left(\begin{array}{c}p \\ p-\bar{s}\end{array}\right)$ Luenberger observers. Each Luenberger observer uses measurements collected from a set of $p-\bar{s}$ sensors. The final output is then selected by exhaustively searching over the outputs of this bank of observers.

\subsection{Exhaustive-Search-Based Observer}

We recall that the states of the attacked system (2.1) can be estimated if and only if, for every subset $\Gamma$ of $\{1, \ldots, p\}$ with at least $p-2 \bar{s}$ elements, the pair $\left(A, C_{\Gamma}\right)$ is observable. We could exploit this result to construct an observer for every set $\Gamma$ with $p-\bar{s}$ elements (which is greater than $p-2 \bar{s}$ ) as follows:

$$
\begin{aligned}
\hat{x}_{\Gamma}^{(t+1)} & =A \hat{x}_{\Gamma}^{(t)}+B u^{(t)}+L_{\Gamma}\left(y_{\Gamma}^{(t)}-\hat{y}_{\Gamma}^{(t)}\right), \\
\hat{y}_{\Gamma}^{(t)} & =C_{\Gamma} \hat{x}_{\Gamma}^{(t)},
\end{aligned}
$$

where $\hat{x}_{\Gamma}$ denotes the state estimate generated from the input $u$ and output $y_{i}, i \in \Gamma . L_{\Gamma}$ can be chosen such that the eigenvalues of $A-L_{\Gamma} C_{\Gamma}$ are strictly within the unit disk since the pair $\left(A, C_{\Gamma}\right)$ is observable. Clearly, since at least one subset of $p-\bar{s}$ sensors is attack-free, we expect the output error dynamics $\left\|Y_{\Gamma}-O_{\Gamma} \hat{x}_{\Gamma}\right\|_{2}$ of at least one of these observers to decay. Our aim would then be to select the state estimate whose estimation error is no worse than the one generated by the attackfree sensors. We refer to this approach as the "exhaustive-search-based observer." The architecture of this observer is summarized in Figure 1.

Such a brute-force observer would have, however, two major disadvantages:

- Memory complexity: Running $\left(\begin{array}{c}p \\ p-\bar{s}\end{array}\right)$ estimators as defined in Equation (3.1), each of which produces an estimate $\hat{x}_{\Gamma_{i}} \in \mathbb{R}^{n}$, results in updating a vector of length $n\left(\begin{array}{c}p \\ p-\bar{s}\end{array}\right)$ at each sample time. This requires an amount of memory that is exponential in the number $p$ of sensors.

-Computational complexity: After producing all the $\left(\begin{array}{c}p \\ p-\bar{s}\end{array}\right)$ estimates, they must still be analyzed to select the best state estimate based on some performance criterion. This analysis further adds to the computational complexity of the estimators.

Our main goal is, therefore, to develop a new, scalable observer architecture that overcomes the disadvantages above.

\subsection{Multi-Modal SMT-Based Observer}

To reduce memory complexity, we propose to replace the bank of $\left(\begin{array}{c}p \\ p-\bar{s}\end{array}\right)$ observers with a single multi-modal Luenberger (MML)-observer which is still able to produce all the estimates of the naive observer. The MML-observer uses the input $u^{(t)}$ and measurements $y^{(t)}$ collected from all the sensors to update an extended state estimate $\hat{z}^{(t)}$. Whenever needed, the extended state 
estimate $\hat{z}^{(t)}$ can be transformed into a state estimate $\hat{x}_{\Gamma}^{(t)}$ that matches the data corresponding to the set of sensors indexed by $\Gamma$. We show in Section 4 that the memory usage for the extended state estimate $\hat{z}^{(t)}$ scales linearly with $p$ as opposed to the exponential scaling of the exhaustivesearch-based observer (3.1).

Although the MML-observer reduces the memory requirements, we would still need to analyze all the $\left(\begin{array}{c}p \\ p-\bar{s}\end{array}\right)$ estimates to detect the attack-free sensors. We harness the underlying combinatorial nature of this problem by leveraging techniques from efficient satisfiability solving. To do so, we reformulate the estimation problem as a satisfiability problem as follows.

First, we recall that there is at least one set of sensors $\Gamma^{\star}$ with cardinality $\left|\Gamma^{\star}\right| \geq p-\bar{s}$ such that all the sensors indexed by this set are attack-free. Then, by Proposition A.2 in the Appendix, we can conclude that the output error $\left\|Y_{i}^{(t)}-O_{i} \hat{x}_{\Gamma^{\star}}^{(t)}\right\|_{2}^{2}$ decays exponentially over time, i.e.,

$$
\left\|Y_{i}^{(t)}-O_{i} \hat{x}_{\Gamma^{\star}}^{(t)}\right\|_{2}^{2} \leq \gamma_{i} \bar{\lambda}^{t}+d_{i}\left(t, \bar{\psi}^{2}, \bar{\mu}^{2}\right), \quad \forall i \in \Gamma^{\star},
$$

where $\gamma_{i}, \bar{\lambda}$, and $d_{i}$ are design parameters independent of the knowledge of the specific set $\Gamma^{\star}$. By defining a binary indicator variable $b_{i} \in \mathbb{B}$ such that $b_{i}=0$ when the $i$ th sensor is attack-free and $b_{i}=1$ otherwise, the problem of constructing a secure Luenberger observer can be formulated as the search for an estimate $\eta^{(t)}=\left(\hat{x}^{(t)}, b^{(t)}\right) \in \mathbb{R}^{n} \times \mathbb{B}^{p}$ such that $\eta^{(t)}=\phi^{(t)} \forall t \in \mathbb{N}$, where $\phi^{(t)}$ is defined as

$$
\phi^{(t)}::=\bigwedge_{i=1}^{p}\left(\neg b_{i}^{(t)} \Rightarrow\left\|Y_{i}^{(t)}-O_{i} \hat{x}^{(t)}\right\|_{2}^{2} \leq \gamma_{i} \bar{\lambda}^{t}+d_{i}\left(t, \bar{\psi}^{2}, \bar{\mu}^{2}\right)\right) \wedge\left(\sum_{i=1}^{p} b_{i}^{(t)} \leq \bar{s}\right) .
$$

The first part of $\phi^{(t)}$ asks for an estimate $\hat{x}^{(t)}$ and an assignment for the attack indicator variables $b^{(t)}=\left(b_{1}^{(t)}, \ldots, b_{p}^{(t)}\right)$ such that the discrepancy between the state estimate and the measured outputs decreases exponentially with time. The second clause requires, instead, that the number of attacked sensors be no greater than $\bar{s}$. As denoted by the time argument in $\phi^{(t)}$, at each time $t$, a new formula must be satisfied.

Following the lazy approach to SMT solving, our architecture uses a pseudo-Boolean ${ }^{2}$ satisfiability (pB-SAT) solver to reason about possible assignments for the Boolean variables $b^{(t)}$. The pB-SAT leverages the David-Putnam-Logemann-Loveland (DPLL) algorithm (Malik and Zhang 2009) to suggest a set of sensors that are attack-free. The sensor choice is then passed to the MMLobserver to transform the extended state estimate $\hat{z}^{(t)}$ into a corresponding state estimate $\hat{x}^{(t)}$, which is used to check the satisfiability of the formula $\phi^{(t)}$. If $\phi^{(t)}$ is not satisfied, the selected estimate (and the related sensor set) is incorrect. The observer will then implement a learning procedure to produce a succinct explanation for the infeasibility, i.e., to highlight which sensors are responsible for it. This conflict-driven learning mechanism is instrumental to speed up the process of detecting and isolating the attacked sensors. The overall architecture is summarized in Figure 2. In the following sections, we give details for each of the two building blocks, i.e., the MML-Observer and the SMT-based conflict-driven learning.

\section{MML OBSERVER}

In this section, we explain how to replace the bank of $\left(\begin{array}{c}p \\ p-\bar{s}\end{array}\right)$ observers (3.1) with only one observer which is able to produce the estimates computed by all those observers.

\footnotetext{
${ }^{2}$ A pseudo-Boolean constraint is a linear constraint over Boolean variables with integer coefficients.
} 


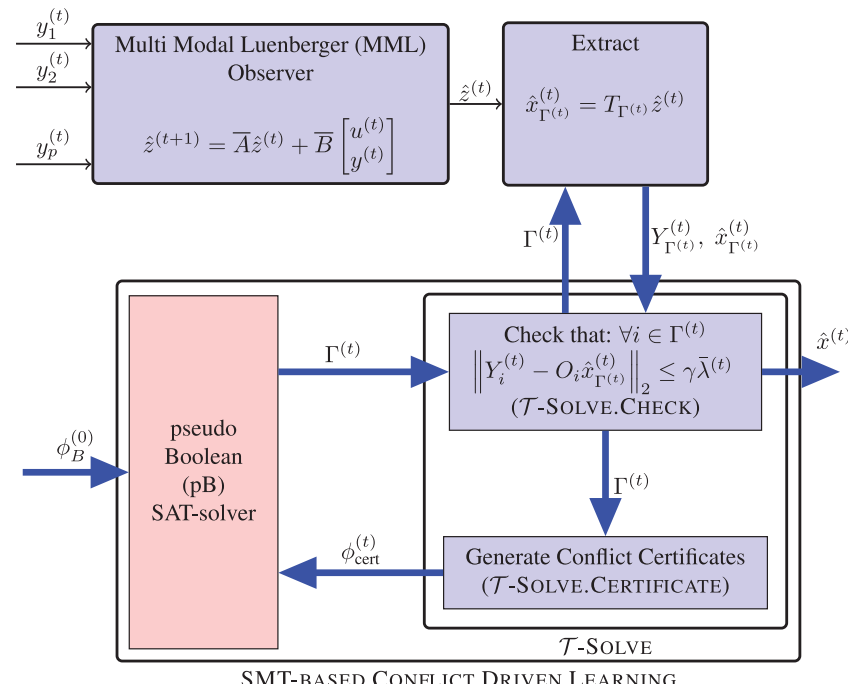

Fig. 2. Architecture of the proposed observer. The observer consists of two main blocks: a Multi-Modal Luenberger $(\mathrm{MML})$ observer that computes an extended estimate $\hat{z}^{(t)}$, which can then be transformed into the state estimate $\hat{x}_{\Gamma}^{(t)}$, for any set $\Gamma$, and an SMT-based conflict-driven learning algorithm that searches for the correct set of sensors $\Gamma$.

Step 1: We start by rewriting the observer (3.1) with initial state $\hat{x}_{\Gamma}^{(0)}=0$ as

$$
\begin{aligned}
\hat{x}_{\Gamma}^{(t+1)} & =\widetilde{A}_{\Gamma} \hat{x}_{\Gamma}^{(t)}+\widetilde{B}_{\Gamma} \widetilde{u}^{(t)}, \quad \hat{x}_{\Gamma}^{(0)}=0, \\
\eta_{\Gamma}^{(t)} & =\hat{x}_{\Gamma}^{(t)},
\end{aligned}
$$

where $\widetilde{A}_{\Gamma}:=A-L_{\Gamma} C_{\Gamma}, \widetilde{B}_{\Gamma}:=\left[B \mathcal{L}_{\Gamma}\right], y^{(t)}:=\left[y_{1}^{(t)} \ldots y_{p}^{(t)}\right]^{\top}$, and $\widetilde{u}^{(t)}:=\left[u^{(t)} y^{(t)}\right]^{\top}$. The columns of $\mathcal{L}_{\Gamma}$ corresponding to the output $y_{i}, i \in \Gamma$, are equal to those of $L_{\Gamma}$ and the other columns are zero.

Step 2: The next step is to choose the observer gain $L_{\Gamma}$ such that $\widetilde{A}_{\Gamma}=A-L_{\Gamma} C_{\Gamma}$ has the same characteristic polynomial:

$$
d(s):=s^{n}+k_{1} s^{n-1}+\cdots+k_{n}
$$

for all $\Gamma$. We note that this step can be always done thanks to the $2 \bar{s}$-sparse observability property.

Step 3: The final step is to find a linear change of coordinates $T_{\Gamma}$ which transforms the observers (4.1) and (4.2) into the following Controllable Canonical Form (CCF) ${ }^{3}$ :

$$
\begin{aligned}
\hat{z}_{\Gamma}^{(t+1)} & =\bar{A} \hat{z}_{\Gamma}^{(t)}+\bar{B} \widetilde{u}^{(t)}, \quad \hat{z}_{\Gamma}^{(0)}=0, \\
\eta_{\Gamma}^{(t)} & =\bar{C}_{\Gamma} \hat{z}_{\Gamma}^{(t)},
\end{aligned}
$$

where $\hat{z}_{\Gamma}^{(t+1)} \in \mathbb{R}^{n \ell}, \ell=m+p$, and

$$
T_{\Gamma} \bar{A}=\widetilde{A}_{\Gamma} T_{\Gamma}, \quad T_{\Gamma} \bar{B}=\widetilde{B}_{\Gamma}, \quad \bar{C}_{\Gamma}=T_{\Gamma},
$$

\footnotetext{
${ }^{3}$ The purpose of using the CCF is to obtain an observer system with a state equation that is independent of the set $\Gamma$ (as shown in Step 3), which allows the multi-observer to be implemented as a family of systems that share a single state equation, but with different output equations.
} 


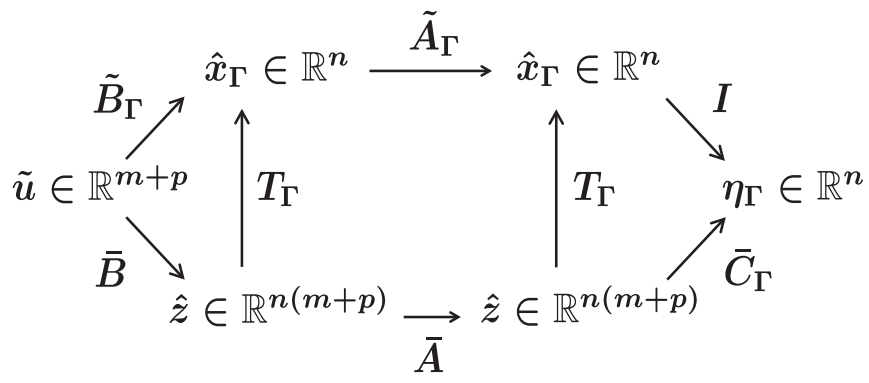

Fig. 3. Commutative diagram of the observers (4.1), (4.2) and (4.7), (4.8).

such that

$$
\bar{A}=\left[\begin{array}{ccccc}
-k_{1} I_{\ell} & -k_{2} I_{\ell} & \ldots & -k_{n-1} I_{\ell} & -k_{n} I_{\ell} \\
I_{\ell} & 0_{\ell} & \ldots & 0_{\ell} & 0_{\ell} \\
0_{\ell} & I_{\ell} & \ldots & 0_{\ell} & 0_{\ell} \\
\vdots & \vdots & \ddots & \vdots & \vdots \\
0_{\ell} & 0_{\ell} & \ldots & I_{\ell} & 0_{\ell}
\end{array}\right], \bar{B}=\left[\begin{array}{c}
I_{\ell} \\
0_{\ell} \\
\vdots \\
0_{\ell} \\
0_{\ell}
\end{array}\right],
$$

where $k_{1}, \ldots, k_{n}$ are the coefficients of the characteristic polynomial (4.3). Note that we dropped the subscript $\Gamma$ from $\bar{A}$ and $\bar{B}$ in Equation (4.4). This follows from step 2 which ensures that all observers, for all sets $\Gamma$, have the same characteristic polynomial and hence they all have the same matrix $\bar{A}$ along with the fact that the definition of the matrix $\bar{B}$ does not depend on the set $\Gamma$.

To find such transformation, we use Proposition 2.3 from De Schutter (2000) on the realization of linear-time-invariant systems to obtain the matrix $T_{\Gamma}$ as

$$
\mathcal{R}_{\Gamma}=\left[\begin{array}{lllll}
\widetilde{B}_{\Gamma} & \widetilde{A}_{\Gamma} \widetilde{B}_{\Gamma} \ldots \widetilde{A}_{\Gamma}^{n-1} \widetilde{B}_{\Gamma}
\end{array}\right], \quad \mathcal{R}^{\prime}=\left[\begin{array}{cccccc}
I_{\ell} & k_{1} I_{\ell} & k_{2} I_{\ell} & \ldots & k_{n-1} I_{\ell} \\
0 \ell & I_{\ell} & k_{1} I_{\ell} & \ldots & k_{n-2} I_{\ell} \\
\vdots & \ddots & \ddots & \ddots & \vdots \\
0 & \ldots & 0_{\ell} & I_{\ell} & k_{1} I_{\ell} \\
0_{\ell} & \ldots & 0_{\ell} & 0 & 0_{\ell} & I_{\ell}
\end{array}\right], \quad T_{\Gamma}=\mathcal{R}_{\Gamma} \mathcal{R}^{\prime} .
$$

Finally, by noticing that all observers are initialized to the same initial condition and they all share the same state update equation, we can rewrite all observers (4.4) and (4.5) as

$$
\begin{aligned}
\hat{z}^{(t+1)} & =\bar{A} \hat{z}^{(t)}+\bar{B} \widetilde{u}^{(t)}, \quad \hat{z}^{(0)}=0, \\
\eta_{\Gamma}^{(t)} & =\bar{C}_{\Gamma} \hat{z}^{(t)},
\end{aligned}
$$

where we dropped the dependency on the set $\Gamma$ in Equation (4.7). In other words, the observer defined by Equations (4.7) and (4.8) updates the intermediate state $\hat{z}^{(t)}$ based on all sensor measurements while still being able to transform $\hat{z}^{(t)}$ in the sensor-dependent output $\hat{x}_{\Gamma}^{(t)}$ for any sensor set $\Gamma$.

Remark 4.1. The size of $\hat{z}$ is $n(m+p)$, i.e., it grows linearly with the number of sensors $p$. This eliminates the need for a state vector that is exponential in $p$ as in the case of the exhaustivesearch-based observer.

The discussion in this section is summarized in Figure 3 and in the following result.

Theorem 4.2. Let the linear dynamical system defined by Equations (2.1) and (2.2) be $2 \bar{s}$-sparse observable. The observer defined by Equations (4.7) and (4.8) generates the same output $\eta_{\Gamma}$ as the 
original bank of observers (4.1) and (4.2) for every input $\tilde{u}$ and for any set of sensors $\Gamma$. Moreover, the size of $\hat{z}$ grows linearly with the number of outputs $p$.

Proof. Since the linear system, defined by Equations (2.1) and (2.2), is $2 \bar{s}$-sparse observable, the pair $\left(A, C_{\Gamma}\right)$ is observable for all $\Gamma$. Hence, we can always choose $L_{\Gamma}$ such that every matrix $\widetilde{A}_{\Gamma}$ satisfies the same characteristic polynomial (4.3). Routine calculations show that

$$
T_{\Gamma} \bar{A}=\widetilde{A}_{\Gamma} T_{\Gamma}, \quad T_{\Gamma} \bar{B}=\widetilde{B}_{\Gamma}, \quad \bar{C}_{\Gamma}=T_{\Gamma},
$$

for all $\Gamma$. Finally, the claim on the size of $\hat{z}$ follows from Remark 4.1.

\section{SMT-BASED ENGINE}

As discussed in Section 3.2, the SMT-based engine has three objectives: (i) hypothesize which sensors are attack-free and hence select the mode of the MML-observer; (ii) check whether the selected set of sensors is, indeed, attack-free; and (iii) generate conflicts (counterexamples) to speed up the search over the possible sensor sets. In this section, we give details on these tasks.

\subsection{Hypothesizing the Attack-Free Sensors}

Searching for the attack-free sensors is combinatorial in nature. At each time instance $t$, we need to select a set $\Gamma^{(t)}$ containing at most $p-\bar{s}$ sensors. To do this, we use the indicator variable $b^{(t)}=$ $\left(b_{1}^{(t)}, \ldots, b_{p}^{(t)}\right) \in \mathbb{B}^{p}$, where we use $b_{i}^{(t)}=0$ to denote that sensor $i$ is considered attack-free at time $t$. At any point in time, the set of hypothesized attack-free sensors $\Gamma^{(t)}$ can then be extracted from $b^{(t)}$ using

$$
\Gamma^{(t)}=\{1, \ldots, p\} \backslash \operatorname{supp}\left(b^{(t)}\right) .
$$

We then ask the PB-SAT-Solve for an assignment over $b_{i}^{(0)}$ (at time $t=0$ ) that satisfies the following constraint:

$$
\phi_{B}^{(0)}:=\sum_{i}^{p} b_{i}^{(0)} \leq \bar{s},
$$

ensuring that at most $\bar{s}$ sensors are regarded as attacked. If the state estimate produced by the MML observer from $\Gamma^{(t)}$ does not satisfy

$$
\left\|Y_{i}-O_{i} \hat{x}_{\Gamma^{(t)}}^{(t)}\right\|_{2}^{2} \leq \gamma_{i} \bar{\lambda}^{t}+d_{i}\left(t, \bar{\psi}^{2}, \bar{\mu}^{2}\right) \quad \forall i \in \Gamma^{(t)},
$$

i.e., it is not bounded by the exponentially decaying envelope, then a new conflict clause is generated by $\mathcal{T}$-Solve.Certificate. This clause takes the form

$$
\phi_{\mathrm{cert}}^{(t)}=\sum_{i \in \mathcal{I}^{(t)}} b_{i}^{(t)} \geq 1
$$

for some set $\mathcal{I}^{(t)} \subseteq \Gamma^{(t)}$, providing a certificate that at least one of the sensors indexed by the set $\mathcal{I}^{(t)}$ is under attack. Details on how to select $\mathcal{I}^{(t)}$ are given in the next subsection. The certificate generated at time $t$ is then conjoined to the formula $\phi_{B}^{(t-1)}$ to create a new Boolean formula $\phi_{B}^{(t)}=$ $\phi_{B}^{(t-1)} \wedge \phi_{\text {cert }}^{(t)}$ that needs to be satisfied by the pB-SAT solver, thus leading to a new candidate set of attack-free sensors. 


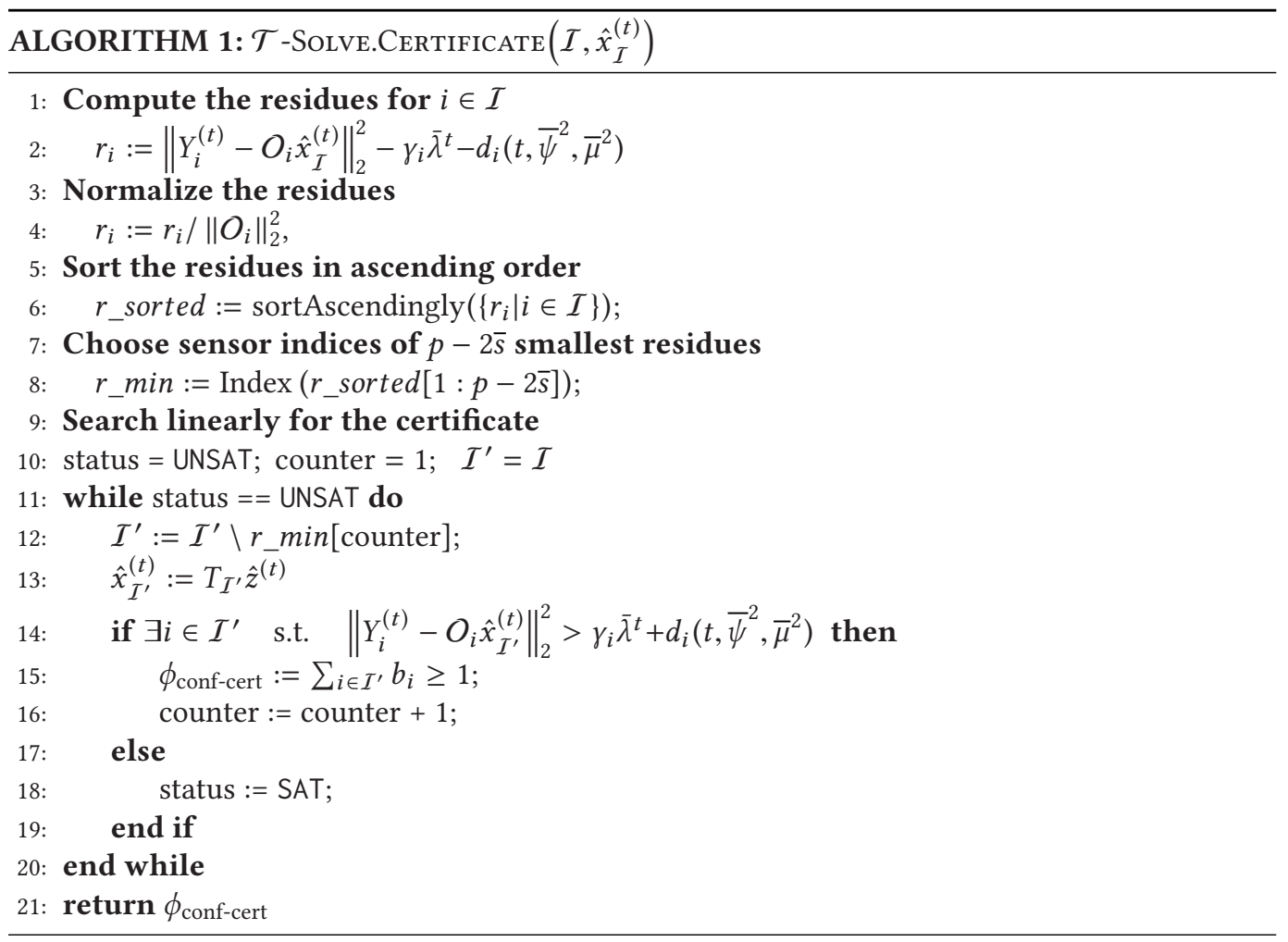

\subsection{Learning a Conflict Clause}

Whenever the set of hypothesized attack-free sensors $\Gamma^{(t)}$ does not satisfy Equation (5.1), we need to generate a compact Boolean certificate that explains the conflict. A trivial certificate could be easily generated as mentioned above:

$$
\phi_{\text {triv-cert }}^{(t)}=\sum_{i \in \Gamma^{(t)}} b_{i}^{(t)} \geq 1,
$$

indicating that at least one of the sensors, which was initially assumed as attack-free (i.e., for which $b_{i}=0$ ), is actually under attack; one of the $b_{i}$ variables should then be set to one in the next assignment of the pB-SAT solver. However, $\phi_{\text {triv-cert }}$ does not provide much information, since it only excludes the current assignment from the search space, and can still lead to exponential execution time (Shoukry et al. 2014; Shoukry et al. 2015). In fact, the generated certificates heavily affect the overall execution time of an SMT solver. Smaller certificates prune the search space faster (Shoukry et al. 2014).

To find such a certificate, we need to search for a subset $\mathcal{I}^{(t)} \subseteq \Gamma^{(t)}$ whose elements cannot all be attack-free. To this end, we start by removing one sensor at a time from the original set and re-run the test (5.1) on the set of remaining sensors. This procedure repeats as long as the residual sensor set is conflicting. Finally, we generate the certificate:

$$
\phi_{\text {conf-cert }}^{(t)}=\sum_{i \in I^{(t)}} b_{i}^{(t)} \geq 1 .
$$



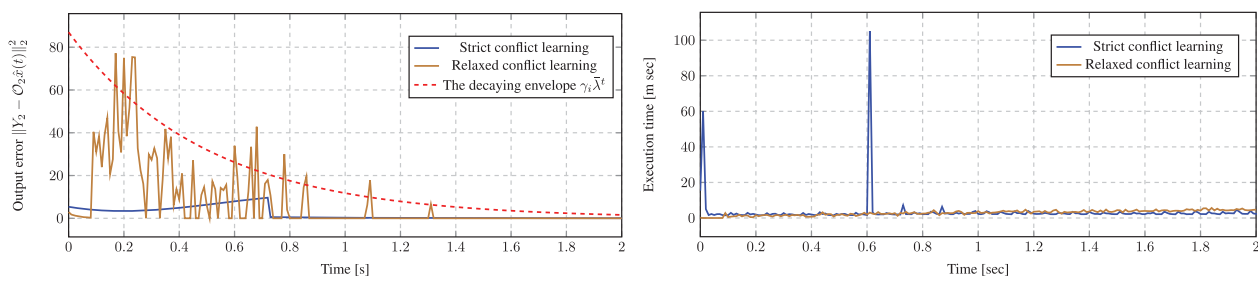

Fig. 4. Difference between Strict and Relaxed conflict learning schemes. Top figure shows the output estimation error $\left\|Y_{i}-O_{i} x\right\|_{2}$ versus the decaying upper bound $\gamma_{i} \bar{\lambda}^{t}$. Bottom figure shows the execution time for both learning schemes.

Termination of the above procedure is guaranteed regardless of the order in which the sensors are chosen. In practice, different orderings may lead to different execution times. In Algorithm 1, we describe a heuristic based on the procedure by Shoukry et al. (2014).

\subsection{Strict versus Relaxed Conflict Clause Learning}

Whenever a set of sensors does not pass the test (5.1), we need to learn a conflict clause and search for a new set of sensors. As the estimation algorithm must run in real time, a natural question is when to terminate the iterative process between hypothesizing a new set of sensors and learning a conflict clause. In particular, we propose two termination schemes, namely, strict conflict clause learning and relaxed conflict clause learning.

In the strict conflict clause learning, as shown in Algorithm 2, new sets of candidate attack-free sets are repetitively generated until we find a set $\Gamma^{(t)}$ that satisfies (5.1). This scheme maintains the following property invariant for all $t$ :

$$
\left\|Y_{i}-O_{i} \hat{x}_{\Gamma^{(t)}}^{(t)}\right\|_{2}^{2} \leq \gamma_{i} \bar{\lambda}^{t}+d_{i}\left(t, \bar{\psi}^{2}, \bar{\mu}^{2}\right), \quad \forall i \in \Gamma^{(t)} .
$$

On the other hand, in the relaxed conflict clause learning in Algorithm 3, a new set of candidate attack-free sensors and conflict clause are generated only once per timestep. We call this scheme "relaxed" since it allows for Equation (5.4) to be violated at some times.

Figure 4 emphasizes the difference between the two learning schemes using the IEEE-14 power bus example discussed in Section 9. As shown in the figure, the norm of the output estimation error $\left\|Y_{i}-O_{i} x\right\|_{2}$ is guaranteed to be always below the decaying bound $\gamma_{i} \bar{\lambda}^{t}$. However, this occasionally comes at the cost of large execution time. The relaxed scheme allows, instead, the output estimation error to exceed the bound but achieves a constant execution time performance, which may be much better than that of the strict observer. In Section 6, we discuss the theoretical guarantees of both the schemes.

\subsection{Scalability-Accuracy Tradeoff}

As shown in the Appendix, the bound $\gamma_{i} \bar{\lambda}^{t}+d_{i}\left(t, \bar{\psi}^{2}, \bar{\mu}^{2}\right)$ used in our algorithms is computed based on the set of sensors that can be under attack in the worst case. This inevitably introduces some conservativeness in the design of the proposed observer, both in terms of convergence speed and error bound on the final estimate, since both $\gamma_{i}$ and $d_{i}$ are computed in the worst case scenario.

Therefore, while the SMT-based engine leads to a significant reduction in time when searching for the attack-free sensors, this advantage possibly comes at the cost of a slower convergence rate and a higher steady-state error when compared with brute-force approaches in which the error bound for each observer in a bank can be independently tuned. We characterize the steady-state estimation error induced by the proposed architecture in the next section. 

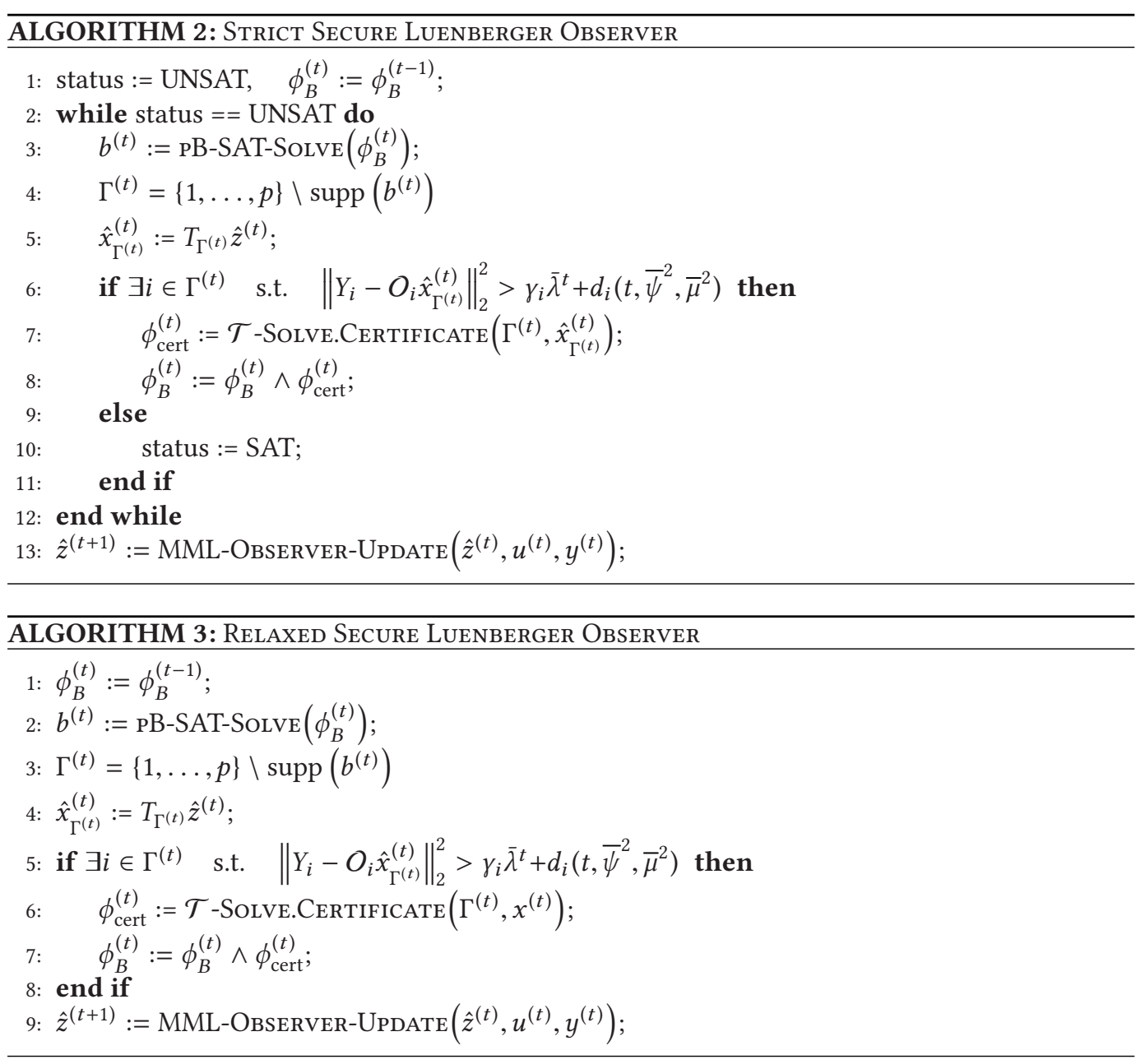

\section{CONVERGENCE ANALYSIS}

We provide proofs of convergence for the two observer architectures introduced in Section 5.3. We start by recalling the following result.

Proposition 6.1 (Proposition II.6 (Shoukry Et AL. 2014)). Let the linear dynamical system defined in Equations (2.1) and (2.2) be $2 \bar{s}$-sparse observable. There exists a $\delta_{2 \bar{s}} \in \mathbb{R}^{+}$such that

$$
0<\delta_{2 \bar{s}} \leq \lambda_{\min }\left\{O_{\Gamma_{2 \bar{s}}}^{T} O_{\Gamma_{2 \bar{s}}}\right\}
$$

for any set $\Gamma_{2 \bar{s}} \subset\{1, \ldots, p\}$ with $\left|\Gamma_{2 \bar{s}}\right| \geq p-2 \bar{s}$.

In the rest of this article, we refer to $\delta_{2 \bar{s}}$ as the $2 \bar{s}$-restricted eigenvalue of the system defined in Equations (2.1) and (2.2). Using this notion of restricted eigenvalue, we can characterize the convergence of the proposed observers.

\subsection{Convergence of the Strict Secure Observer}

THEOREM 6.2. Let the linear dynamical system defined in Equations (2.1) and (2.2) be $2 \bar{s}$-sparse observable. There exist constants $0<\bar{\lambda}<1, \kappa \in \mathbb{R}^{+}$, and $\rho_{t}\left(\bar{\psi}^{2}, \bar{\mu}^{2}\right)$ such that the state estimation error 
$\left\|x^{(t)}-\hat{x}^{(t)}\right\|_{2}$ produced by the secure Luenberger Observer defined in Algorithm 2 at time $t$ is bounded from above as

$$
\left\|x^{(t)}-\hat{x}^{(t)}\right\|_{2}^{2} \leq \kappa \bar{\lambda}^{t}+\rho_{t}\left(\bar{\psi}^{2}, \bar{\mu}^{2}\right) .
$$

Moreover, the state estimation error satisfies

$$
\limsup _{t \rightarrow \infty}\left\|x^{(t)}-\hat{x}^{(t)}\right\|_{2}^{2} \leq \rho\left(\bar{\psi}^{2}, \bar{\mu}^{2}\right),
$$

where $\rho\left(\bar{\psi}^{2}, \bar{\mu}^{2}\right)=\lim _{t \rightarrow \infty} \rho_{t}\left(\bar{\psi}^{2}, \bar{\mu}^{2}\right)$ is given by

$$
\rho\left(\bar{\psi}^{2}, \bar{\mu}^{2}\right)=\frac{1}{\delta_{2 \bar{s}}}\left(\bar{\Psi}+\sqrt{\frac{\alpha}{1-\bar{\lambda}}+\beta}\right)^{2}, \alpha=\sum_{i=1}^{p} \alpha_{i}, \beta=\sum_{i=1}^{p} \beta_{i},
$$

and $\alpha_{i}, \beta_{i}$, and $\bar{\lambda}$ are as defined in Proposition A.2.

Proof. It follows from the $2 \bar{s}$-sparse observability and Proposition A.2 in the Appendix that there exist observer gains such that the error dynamics of the attack-free observer is stable. In other words, there exist constants $\gamma_{i}, 0<\bar{\lambda}<1, d_{i}\left(t, \bar{\psi}^{2}, \bar{\mu}^{2}\right)$ and sets $\Gamma^{(t)}$ such that Equation (5.1) holds. Hence, we conclude that the following inequality holds:

$$
\left\|Y_{i}-O_{i} \hat{x}_{\Gamma^{(t)}}^{(t)}\right\|_{2}^{2} \leq \gamma_{i} \bar{\lambda}^{t}+d_{i}\left(t, \bar{\psi}^{2}, \bar{\mu}^{2}\right), \quad \forall i \in \Gamma^{(t)} \forall t \in \mathbb{N} .
$$

We now define $\mathcal{I}^{(t)}=\Gamma^{(t)} \backslash \operatorname{supp}\left(E^{(t)}\right)$, and $\mathcal{I}^{\prime(t)}=\operatorname{supp}\left(E^{(t)}\right)$. Then, the following holds:

$$
\begin{aligned}
\left\|Y_{\Gamma^{(t)}}^{(t)}-O_{\Gamma^{(t)}} \hat{x}^{(t)}\right\|_{2}^{2} & =\left\|O_{\Gamma^{(t)}}\left(x^{(t)}-\hat{x}^{(t)}\right)+E_{\Gamma^{(t)}}^{(t)}+\Psi_{\Gamma^{(t)}}^{(t)}\right\|_{2}^{2} \\
& =\left\|O_{I^{(t)}}\left(x^{(t)}-\hat{x}^{(t)}\right)+\Psi_{I^{(t)}}^{(t)}\right\|_{2}^{2}+\left\|O_{I^{\prime}(t)}\left(x^{(t)}-\hat{x}^{(t)}\right)+E_{I^{\prime}(t)}^{(t)}+\Psi_{I^{\prime}(t)}^{(t)}\right\|_{2}^{2} \\
& \stackrel{(a)}{\geq}\left\|O_{I^{(t)}}\left(x^{(t)}-\hat{x}^{(t)}\right)+\Psi_{I^{(t)}}^{(t)}\right\|_{2}^{2} \stackrel{(b)}{\geq}\left(\left\|O_{I^{(t)}}\left(x^{(t)}-\hat{x}^{(t)}\right)\right\|_{2}-\left\|\Psi_{I^{(t)}}^{(t)}\right\|_{2}\right)^{2},
\end{aligned}
$$

where inequality $(a)$ follows from the fact that the set $\mathcal{I}^{(t)}$ contains all attack-free sensors and hence $E_{I^{(t)}}^{(t)}$ is equal to zero while inequality $(b)$ follows from the reverse triangular inequality which states that $\|a+b\|_{2} \geq\left|\|a\|_{2}-\|b\|_{2}\right|$. Combining Equations (6.1) and (6.2) we conclude that

$$
\begin{aligned}
& \left(\left\|O_{I^{(t)}}\left(x^{(t)}-\hat{x}^{(t)}\right)\right\|_{2}-\left\|\Psi_{I^{(t)}}^{(t)}\right\|_{2}\right)^{2} \stackrel{(c)}{\leq} \sum_{i \in \Gamma^{(t)}}\left(\gamma_{i} \bar{\lambda}^{t}+d_{i}\left(t, \bar{\psi}^{2}, \bar{\mu}^{2}\right)\right) \stackrel{(d)}{\leq} \gamma \bar{\lambda}^{t}+d\left(t, \bar{\psi}^{2}, \bar{\mu}^{2}\right) \\
& \Rightarrow\left\{\begin{array}{l}
\left\|O_{I^{(t)}}\left(x^{(t)}-\hat{x}^{(t)}\right)\right\|_{2} \geq\left\|\Psi_{I^{(t)}}^{(t)}\right\|_{2}-\sqrt{\gamma \bar{\lambda}^{t}+d\left(t, \bar{\psi}^{2}, \bar{\mu}^{2}\right)} \\
\left\|O_{I^{(t)}}\left(x^{(t)}-\hat{x}^{(t)}\right)\right\|_{2} \leq\left\|\Psi_{I^{(t)}}^{(t)}\right\|_{2}+\sqrt{\gamma \bar{\lambda}^{t}+d\left(t, \bar{\psi}^{2}, \bar{\mu}^{2}\right)}
\end{array}\right. \\
& \Rightarrow\left\{\begin{array}{l}
\left\|O_{I^{(t)}}\left(x^{(t)}-\hat{x}^{(t)}\right)\right\|_{2}^{2} \geq\left(\left\|\Psi_{I^{(t)}}^{(t)}\right\|_{2}-\sqrt{\gamma \bar{\lambda}^{t}+d\left(t, \bar{\psi}^{2}, \bar{\mu}^{2}\right)}\right)^{2} \\
\left\|O_{I^{(t)}}\left(x^{(t)}-\hat{x}^{(t)}\right)\right\|_{2}^{2} \leq\left(\left\|\Psi_{I^{(t)}}^{(t)}\right\|_{2}+\sqrt{\gamma^{2} \bar{\lambda}^{t}+d\left(t, \bar{\psi}^{2}, \bar{\mu}^{2}\right)}\right)^{2},
\end{array}\right.
\end{aligned}
$$

where inequality (c) follows from Equation (6.1), which ensures that Algorithm 2 always returns an estimate $\eta^{(t)}=\left(\hat{x}^{(t)}, b^{(t)}\right)$ that satisfies $\phi^{(t)}$ for all $t$. Inequality $(d)$ is, instead, obtained by setting $\gamma=\sum_{i=1}^{p} \gamma_{i}$ and $d\left(t, \bar{\psi}^{2}, \bar{\mu}^{2}\right)=\sum_{i=1}^{p} d_{i}\left(t, \bar{\psi}^{2}, \bar{\mu}^{2}\right)$. Since we are interested in an upper bound for the error, we only focus on the second inequality in Equation (6.3).

We now observe that, because the attacker can corrupt at most $\bar{s}$ sensors, the cardinality of $\operatorname{supp}\left(E^{(t)}\right)$ is bounded by $\bar{s}$, i.e., $\left|\operatorname{supp}\left(E^{(t)}\right)\right| \leq \bar{s}$. Moreover, because the pB-SAT solver assumes at 
most that $\bar{s}$ sensors can be under attack, then the set $\Gamma^{(t)}=\{1, \ldots, p\} \backslash \operatorname{supp}\left(b^{(t)}\right)$ has a cardinality bounded by $p-\bar{s}$, i.e., $\left|\Gamma^{(t)}\right| \geq p-\bar{s}$. Using these two facts we conclude that the cardinality of $\mathcal{I}^{(t)}=\Gamma^{(t)} \backslash \operatorname{supp}\left(E^{(t)}\right)$ is bounded by $p-2 \bar{s}$, i.e., $\left|\mathcal{I}^{(t)}\right| \geq p-2 \bar{s}$. By using Equation (6.3), we then conclude

$$
\begin{aligned}
& \left\|O_{I^{(t)}}\left(x^{(t)}-\hat{x}^{(t)}\right)\right\|_{2}^{2} \leq\left(\left\|\Psi_{I^{(t)}}^{(t)}\right\|_{2}+\sqrt{\gamma \bar{\lambda}^{t}+d\left(t, \bar{\psi}^{2}, \bar{\mu}^{2}\right)}\right)^{2} \\
& \Rightarrow \delta_{2 \bar{s}}\left\|x^{(t)}-\hat{x}^{(t)}\right\|_{2}^{2} \leq\left(\left\|\Psi_{I^{(t)}}^{(t)}\right\|_{2}+\sqrt{\gamma \bar{\lambda}^{t}+d\left(t, \bar{\psi}^{2}, \bar{\mu}^{2}\right)}\right)^{2} \\
& \Rightarrow\left\|x^{(t)}-\hat{x}^{(t)}\right\|_{2}^{2} \leq \frac{1}{\delta_{2 \bar{s}}}\left(\left\|\Psi_{I^{(t)}}^{(t)}\right\|_{2}+\sqrt{\gamma \bar{\lambda}^{t}+d\left(t, \bar{\psi}^{2}, \bar{\mu}^{2}\right)}\right)^{2} \leq \frac{2}{\delta_{2 \bar{s}}} \bar{\Psi}^{2}+\frac{2}{\delta_{2 \bar{s}}}\left(\gamma \bar{\lambda}^{t}+d\left(t, \bar{\psi}^{2}, \bar{\mu}^{2}\right)\right) .
\end{aligned}
$$

The result stated in the theorem then holds with $\kappa=\frac{2 \gamma}{\delta_{2 \bar{s}}}$ and $\rho_{t}\left(\bar{\psi}^{2}, \bar{\mu}^{2}\right)=\frac{2}{\delta_{2 \bar{s}}}\left(\bar{\Psi}^{2}+d\left(t, \bar{\psi}^{2}\right.\right.$, $\left.\left.\bar{\mu}^{2}\right)\right)$.

\subsection{Convergence of the Relaxed Secure Observer}

Theorem 6.3. Let the linear dynamical system defined in Equations (2.1) and (2.2) be $2 \bar{s}$-sparse observable. The state estimation error produced by the secure Luenberger Observer defined in Algorithm 3 satisfies

$$
\limsup _{t \rightarrow \infty}\left\|x^{(t)}-\hat{x}^{(t)}\right\|_{2}^{2} \leq \rho\left(\bar{\psi}^{2}, \bar{\mu}^{2}\right) .
$$

Proof. It follows from the $2 \bar{s}$-sparse observability and Proposition A. 2 in the Appendix that there exist observer gains such that the error dynamics of the attack-free observer is stable. Therefore, there exist constants $\gamma_{i}, 0<\bar{\lambda}<1, d_{i}\left(t, \bar{\psi}^{2}, \bar{\mu}^{2}\right)$ and sets $\Gamma^{(t)}$ such that Equation (5.1) holds. However, since Algorithm 3 may terminate before finding such $\Gamma^{(t)}$, the condition (5.1) may not always hold in the case of the relaxed observer.

On the other hand, we note that (i) there are finitely many choices for the set $\Gamma^{(t)}$ since the indicator Boolean variable $b^{(t)}$ can take at most a finite number of values; (ii) whenever a certificate $\phi_{\text {conf-cert }}^{(t)}$ is learnt, at least one assignment of $b^{(t)}$ is ruled out of the search space. Therefore, there exists a time $t^{\prime}$ such that the following holds:

$$
\left\|Y_{i}-O_{i} \hat{x}_{\Gamma^{(t)}}^{(t)}\right\|_{2}^{2} \leq \gamma_{i} \bar{\lambda}^{t}+d_{i}\left(t, \bar{\psi}^{2}, \bar{\mu}^{2}\right), \quad \forall i \in \Gamma^{(t)} \forall t \geq t^{\prime} .
$$

We can the follow the same line of reasoning as in the proof of Theorem 6.2 starting with time $t^{\prime}$ to conclude on the convergence of the relaxed scheme.

\section{ROBUST OBSERVER DESIGN}

As shown in Section 6, whenever noise is present, the state estimation error converges asymptotically (with rate equal to $\bar{\lambda}$ ) to some positive constant $\rho$. A closer look at this constant, as apparent from the definitions in Proposition A.2, shows its dependency on the norm of the observer gains $\left\|L_{\Gamma}\right\|_{2}$, introduced in Equation (3.1). There is a tradeoff between noise insensitivity and convergence performance of the proposed observer design. Observers with higher gains, i.e., higher $\left\|L_{\Gamma}\right\|_{2}$, lead to amplification of sensor noise, which results in a fast convergence rate but at the cost of larger $\rho$ and poorer state estimates. ${ }^{4}$ On the contrary, observers with lower gains, i.e., lower $\left\|L_{\Gamma}\right\|_{2}$, may result in slower convergence but better state estimates. In this subsection, we discuss

\footnotetext{
${ }^{4}$ The process noise $\mu^{(t)}$ appears as an additive term in the observer equations and is not amplified by the observer gain $L$.
} 
how to design the parameters of the proposed observer architecture to achieve an "optimal" state estimation error while maintaining convergence guarantees.

A common approach for designing robust observers is to search for an optimal gain by casting an optimization problem subject to Linear Matrix Inequality (LMI) constraints. In such a formulation, the objective function captures the noise performance to be maximized, while the LMI constraints guarantee stability in the Lyapunov sense, hence convergence of the state estimate. However, in our case, such an approach would lead to a large optimization problem that would be tedious to assemble and computationally challenging. In fact, as described in Section 4, we are interested in selecting one gain $L_{\Gamma}$ for each sensor subset $\Gamma$ subject to the constraint that all the resulting $A-L_{\Gamma} C_{\Gamma}$ observers share the same characteristic polynomial. Finding all these optimal gains with a conventional approach would require a joint optimization problem including one LMI for each observer, for a total of $\left(\begin{array}{c}p \\ p-\bar{s}\end{array}\right)$ LMI constraints. To avoid dealing with such a large size, we propose to follow, instead, a different procedure, which does not explicitly design the observer gains $L_{\Gamma}$ but focuses on the design of the MML observer directly from Equation (4.7).

Based on the structure of the MML observer in CCF from Equation (4.7), we observe that we can reduce the problem of designing robust estimators to that of finding an "optimal" assignment for the parameters $\left(k_{1}, \ldots, k_{n}\right)$. To do this, while leveraging the existing LMI-based tools for observer design, we re-write Equation (4.7) as

$$
\begin{aligned}
& \hat{z}^{(t+1)}=\underbrace{\left[\begin{array}{ccccc}
-k_{1} I_{\ell} & -k_{2} I_{\ell} & \ldots & -k_{n-1} I_{\ell} & -k_{n} I_{\ell} \\
I_{\ell} & 0_{\ell} & \ldots & 0_{\ell} & 0_{\ell} \\
0_{\ell} & I_{\ell} & \ldots & 0_{\ell} & 0_{\ell} \\
\vdots & \vdots & \ddots & \vdots & \vdots \\
0_{\ell} & 0_{\ell} & \ldots & I_{\ell} & 0_{\ell}
\end{array}\right]}_{\bar{A}} \hat{z}^{(t)}+\underbrace{\left[\begin{array}{c}
I_{\ell} \\
0_{\ell} \\
\vdots \\
0_{\ell} \\
0_{\ell}
\end{array}\right]}_{\bar{B}} \underbrace{\left[\begin{array}{c}
u^{(t)} \\
y_{1}^{(t)} \\
\vdots \\
y_{p}^{(t)}
\end{array}\right]}_{\widetilde{u}} \\
& =(\underbrace{\left[\begin{array}{ccccc}
0_{\ell} & 0_{\ell} & \ldots & 0_{\ell} & 0_{\ell} \\
I_{\ell} & 0_{\ell} & \ldots & 0_{\ell} & 0_{\ell} \\
0_{\ell} & I_{\ell} & \ldots & 0_{\ell} & 0_{\ell} \\
\vdots & \vdots & \ddots & \vdots & \vdots \\
0_{\ell} & 0_{\ell} & \ldots & I_{\ell} & 0_{\ell}
\end{array}\right]}_{\Lambda}-\underbrace{\left[\begin{array}{ccccc}
I_{\ell} & I_{\ell} & \ldots & I_{\ell} & I_{\ell} \\
0_{\ell} & 0_{\ell} & \ldots & 0_{\ell} & 0_{\ell} \\
0_{\ell} & 0_{\ell} & \ldots & 0_{\ell} & 0_{\ell} \\
\vdots & \vdots & \ddots & \vdots & \vdots \\
0_{\ell} & 0_{\ell} & \ldots & 0_{\ell} & 0_{\ell}
\end{array}\right]}_{M} \underbrace{\left[\begin{array}{cccccc}
k_{1} I_{\ell} & 0_{\ell} & \ldots & 0_{\ell} & 0_{\ell} \\
0_{\ell} & k_{2} I_{\ell} & & 0_{\ell} & 0_{\ell} \\
\vdots & \vdots & \ddots & \vdots & \vdots \\
0 & 0_{\ell} & \ldots & k_{n-1} I_{\ell} & 0_{\ell} \\
0_{\ell} & 0_{\ell} & \ldots & 0_{\ell} & k_{n} I_{\ell}
\end{array}\right]}_{K}) \hat{z}^{(t)} \\
& +\underbrace{\left[\begin{array}{c}
I_{\ell} \\
0_{\ell} \\
\vdots \\
0_{\ell} \\
0_{\ell}
\end{array}\right]}_{\bar{B}} \underbrace{\left[\begin{array}{c}
u^{(t)} \\
C_{1} x^{(t)}+a_{1}^{(t)} \\
\vdots \\
C_{p} x^{(t)}+a_{p}^{(t)}
\end{array}\right]}_{v^{(t)}}+\underbrace{\left[\begin{array}{c}
I_{\ell} \\
0_{\ell} \\
\vdots \\
0_{\ell} \\
0_{\ell}
\end{array}\right]\left[\begin{array}{c}
0_{m \times p} \\
I_{p \times p}
\end{array}\right]}_{N} \underbrace{\left[\begin{array}{c}
\psi_{1}^{(t)} \\
\vdots \\
\psi_{p}^{(t)}
\end{array}\right]}_{\psi^{(t)}} \\
& =(\Lambda-M K) \hat{z}^{(t)}+\bar{B} v^{(t)}+N \psi^{(t)} .
\end{aligned}
$$

Moreover, because of the linearity of the observer, to characterize the effect of sensor noise on the state estimate, we can assume, without loss of generality, that the state $x^{(t)}$, the input $u^{(t)}$, the attack $a^{(t)}$, and the process noise $\mu^{(t)}$ are all equal to zero for all $t$. Under this assumption, Equation (7.1) reduces to

$$
\hat{z}^{(t+1)}=(\Lambda-M K) \hat{z}^{(t)}+N \psi^{(t)} .
$$


Our objective is then to design the matrix $K$ in Equation (7.1), which we compactly denote as $\operatorname{diag}\left(k_{1} I_{\ell}, \ldots, k_{n} I_{\ell}\right)$, to attenuate the effect of the noise $\psi^{(t)}$ on the state estimate while ensuring convergence. To formalize this problem, we make the additional assumption that the noise signal $\psi^{(t)}$ is energy bounded, i.e., $\|\psi\|_{l^{2}}<\infty$ holds, where $\|\psi\|_{l^{2}}:=\sqrt{\sum_{t=0}^{\infty}\left\|\psi^{(t)}\right\|_{2}}$ is the $l^{2}$-norm of $\psi^{(t)}$. We then introduce an attenuation factor $\sigma$ for the effect of noise on the state estimate, and cast the design problem as an optimization problem as follows.

Problem 7.1 (Robust Luenberger Observer Design). Consider the MML observer defined in Equaation (7.2). For energy bounded noise $\psi$, find the scalars $\left(k_{1}, \ldots, k_{n}\right)$ and the attenuation factor $\sigma$ that solve the following optimization problem:

$$
\begin{array}{ll}
\min _{k_{1}, \ldots, k_{n}, \sigma} & \sigma \\
\text { subject to: } & \left|\lambda_{i}\{\Lambda-M K\}\right|<1 \quad \forall i \in\{1, \ldots, n \ell\} \quad \text { and } \quad\|\hat{z}\|_{l^{2}} \leq \sigma\|\psi\|_{l^{2}},
\end{array}
$$

where $K=\operatorname{diag}\left(k_{1} I_{\ell}, \ldots, k_{n} I_{\ell}\right)$.

To tackle the above problem, we start by invoking the Bounded Real Lemma (de Souza and Xie 1992) as follows.

Proposition 7.2. Consider the MML observer defined in Equation (7.2). For a given matrix $K$ and attenuation factor $\sigma$, the constraints (7.3) hold if and only if there exists a positive-definite matrix $P=P^{T}>0$ such that

$$
\left[\begin{array}{cccc}
P & 0 & (\Lambda-M K)^{T} P & I \\
* & \sigma I & N^{T} P & 0 \\
* & * & P & 0 \\
* & * & * & \sigma I
\end{array}\right]>0 .
$$

Using Proposition 7.2, we can rewrite Problem 7.1 as follows.

Problem 7.3 (Robust Observer Design Problem with Bilinear Matrix Inequality).

$$
\begin{aligned}
& \min _{k_{1}, \ldots, k_{n}, \sigma, P^{T}>0} \sigma \\
& \text { subject to: }\left\{\left[\begin{array}{llcc}
P & 0 & (\Lambda-M K)^{T} P & I \\
* & \sigma I & N^{T} P & 0 \\
* & * & P & 0 \\
* & * & * & \sigma I
\end{array}\right]>0 .\right. \\
& K=\operatorname{diag}\left(k_{1} I_{\ell}, \ldots, k_{n} I_{\ell}\right) .
\end{aligned}
$$

Unfortunately, because the product of $K$ and $P$ appears in the matrix constraint (7.4), the matrix inequality is a bilinear inequality, the optimization problem above is non-convex, and may not be efficiently solvable. Therefore, in the rest of this section, we discuss two algorithms that can be used to provide sub-optimal solutions to Problem 7.3 in an efficient way, using an off-the-shelf convex optimization solver.

\subsection{Robust Observer Design Using Cone Complementary Linearization}

To circumvent the bilinear terms in the matrix inequality (7.4), we define the change of variables $Q=P^{-1}$ and apply the congruence transformation with transformation matrix $\operatorname{diag}(I, I, Q, I)$ to 
the left-hand side of Equation (7.4), thus obtaining

$$
\begin{aligned}
& {\left[\begin{array}{cccc}
P & 0 & (\Lambda-M K)^{T} P & I \\
* & \sigma I & N^{T} P & 0 \\
* & * & P & 0 \\
* & * & * & \sigma I
\end{array}\right]>0} \\
& \Leftrightarrow\left[\begin{array}{cccc}
I & 0 & 0 & 0 \\
* & I & 0 & 0 \\
* & * & Q & 0 \\
* & * & * & I
\end{array}\right]\left[\begin{array}{cccc}
P & 0 & (\Lambda-M K)^{T} P & I \\
* & \sigma I & N^{T} P & 0 \\
* & * & P & 0 \\
* & * & * & \sigma I
\end{array}\right]\left[\begin{array}{cccc}
I & 0 & 0 & 0 \\
* & I & 0 & 0 \\
* & * & Q & 0 \\
* & * & * & I
\end{array}\right]>0 \\
& \Leftrightarrow\left[\begin{array}{cccc}
P & 0 & (\Lambda-M K)^{T} & I \\
* & \sigma I & N^{T} & 0 \\
* & * & Q & 0 \\
* & * & * & \sigma I
\end{array}\right]>0 .
\end{aligned}
$$

Thus, the bilinear matrix inequality (7.4) holds if and only if the there exist positive-definite matrices $P$ and $Q$ such that the linear matrix inequality (7.5) holds with $P Q=I$. This can be further written as

$$
\left[\begin{array}{cccc}
P & 0 & (\Lambda-M K)^{T} & I \\
* & \sigma I & N^{T} & 0 \\
* & * & Q & 0 \\
* & * & * & \sigma I
\end{array}\right]>0, \quad\left[\begin{array}{cc}
P & I \\
I & Q
\end{array}\right]>0, \quad \operatorname{trace}(P Q)=n(m+p)=n \ell .
$$

The constraints in Equation (7.6) are equivalent to the sufficient and necessary condition in Proposition 7.2. This suggests the following iterative algorithm for solving Problem 7.3. Starting with $\sigma=0$, we check the feasibility of the constraint (7.4) by solving the following optimization problem.

Problem 7.4 (Optimization Problem for CCL-Based Design).

$$
\begin{aligned}
& \min _{k_{1}, \ldots, k_{n}, P=P^{T}>0, Q=Q^{T}>0} \quad \operatorname{trace}(P Q)
\end{aligned}
$$

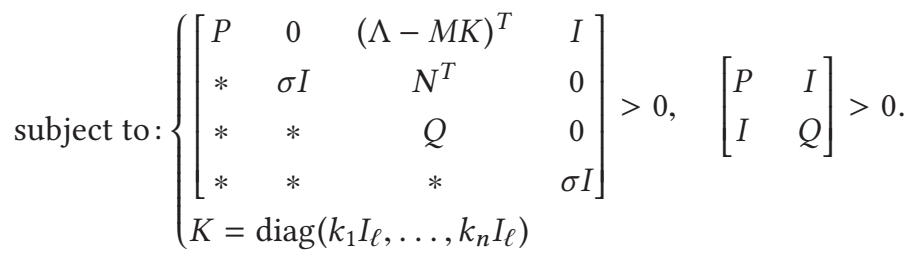

This nonlinear optimization problem (Problem 7.4) can be solved efficiently using the Cone Complementary Linearization (CCL) algorithm (El Ghaoui et al. 1997). The next step is to check whether the resulting trace $\left(P^{*} Q^{*}\right)$ is equal to $n \ell$. If this is not the case, then we conclude that the current choice of $\sigma$ is not feasible, and we invoke the same trace minimization problem with a higher value of $\sigma$, i.e., $\sigma+\epsilon$ for some small enough $\epsilon \in \mathbb{R}^{+}$, until a feasible value of $\sigma$ is obtained. This procedure is summarized in Algorithm 4. The properties of Algorithm 4 are expressed by the following result whose proof follows directly from the previous discussion.

Theorem 7.5. Consider the MML observer defined in (7.2). If there exist scalars $k_{1}, \ldots, k_{n}, \sigma>0$, and positive definite matrices $P=P^{T}>0$ and $Q=Q^{T}>0$ such that Problem 7.4 is feasible, then the bilinear constraint (7.4) is also feasible. Therefore, a solution of Algorithm 4 is a feasible solution for Problem 7.3, hence for Problem 7.1. 


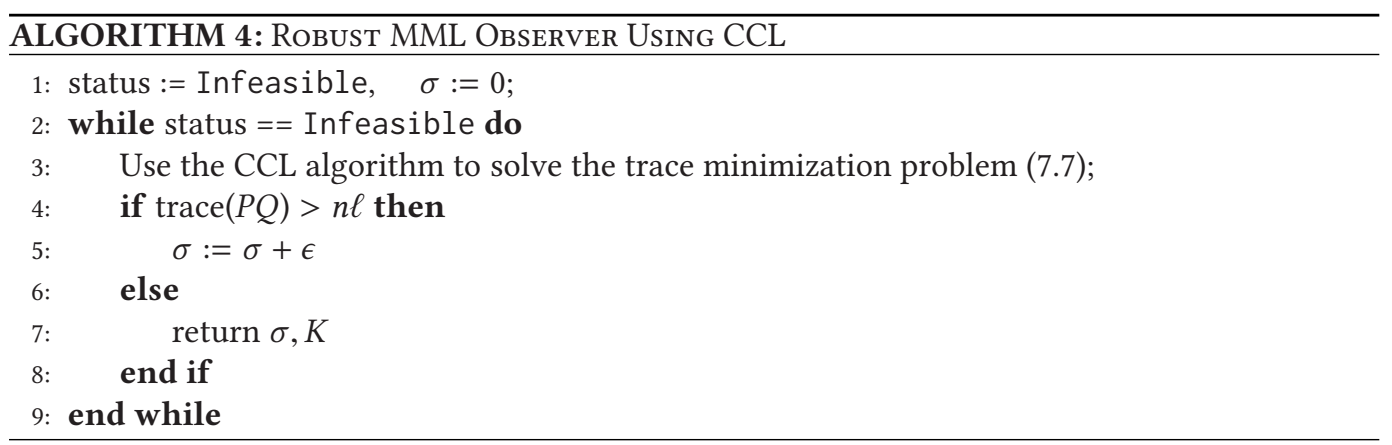

\subsection{Robust Observer Design Using Structured Slack Matrix}

A major drawback of the CCL-based method above stems from the fact that it cannot simultaneously search for the attenuation factor $\sigma$ and the observer gain matrix $K$, thus invoking the CCL algorithm multiple times. To overcome this problem, in this subsection, we propose a relaxation of the bilinear inequality (7.4) that allows casting Problem 7.3 as a convex constrained problem which searches for both $\sigma$ and $K$ simultaneously.

We also consider in this case the change of variables $Q=P^{-1}$ and, for any invertible matrix $G$, the following congruence transformation of the left-hand side of the matrix constraint (7.4):

$$
\begin{aligned}
& {\left[\begin{array}{cccc}
P & 0 & (\Lambda-M K)^{T} P & I \\
* & \sigma I & N^{T} P & 0 \\
* & * & P & 0 \\
* & * & * & \sigma I
\end{array}\right]>0} \\
& \Leftrightarrow\left[\begin{array}{cccc}
G^{T} & 0 & 0 & 0 \\
* & I & 0 & 0 \\
* & * & Q & 0 \\
* & * & * & I
\end{array}\right]\left[\begin{array}{cccc}
P & 0 & (\Lambda-M K)^{T} P & I \\
* & \sigma I & N^{T} P & 0 \\
* & * & P & 0 \\
* & * & * & \sigma I
\end{array}\right]\left[\begin{array}{cccc}
G & 0 & 0 & 0 \\
* & I & 0 & 0 \\
* & * & Q & 0 \\
* & * & * & I
\end{array}\right]>0 \\
& \Leftrightarrow\left[\begin{array}{cccc}
G^{T} P G & 0 & G^{T}(\Lambda-M K)^{T} & G^{T} \\
* & \sigma I & N^{T} & 0 \\
* & * & Q & 0 \\
* & * & * & \sigma I
\end{array}\right]>0 \\
& \stackrel{(a)}{\Leftarrow}\left[\begin{array}{cccc}
G^{T}+G-Q & 0 & G^{T}(\Lambda-M K)^{T} & G^{T} \\
* & \sigma I & N^{T} & 0 \\
* & * & Q & 0 \\
* & * & * & \sigma I
\end{array}\right]>0,
\end{aligned}
$$

where (a) follows from the fact that $G^{T} P G=G^{T} Q G>G^{T}+G-Q$. The inequality (7.8) is still bilinear because of the term $G^{T}(\Lambda-M K)^{T}$. To reduce Equation (7.8) to a linear matrix inequality, we resort to a matrix $G$ with a fixed structure of the form

$$
G=\operatorname{diag}\left(g_{1} I_{\ell}, \ldots, g_{n} I_{\ell}\right),
$$

$g_{1}, \ldots, g_{\ell}$ being scalar terms. We can then write the bilinear term $G^{T}(\Lambda-M K)^{T}$ as

$$
(\Lambda-M K) G=\Lambda G-M H,
$$

where $H=\left(h_{1} I_{\ell}, \ldots, h_{n} I_{\ell}\right)$ and $h_{i}=g_{i} k_{i}$ for all $i \in\{1, \ldots, \ell\}$. Therefore, if there exist a positivedefinite matrix $P$ and block diagonal matrices $G$ and $H$ such that the LMI 


$$
\left[\begin{array}{cccc}
G^{T}+G-Q & 0 & G^{T} \Lambda^{T}-H^{T} M^{T} & G^{T} \\
* & \sigma I & N^{T} & 0 \\
* & * & Q & 0 \\
* & * & * & \sigma I
\end{array}\right]>0
$$

is feasible, then we conclude that the matrix inequality (7.4) is also feasible with $P=Q^{-1}$ and $K=H G^{-1}$. Note that the feasibility of Equation (7.9) is just a sufficient condition for the feasibility of Equation (7.4) due to the imposed structure on the matrix $G$. This suggests that the bilinear (nonconvex) optimization Problem 7.3 can be relaxed to the following convex optimization problem.

Problem 7.6 (Optimization Problem for SSM-Based Design).

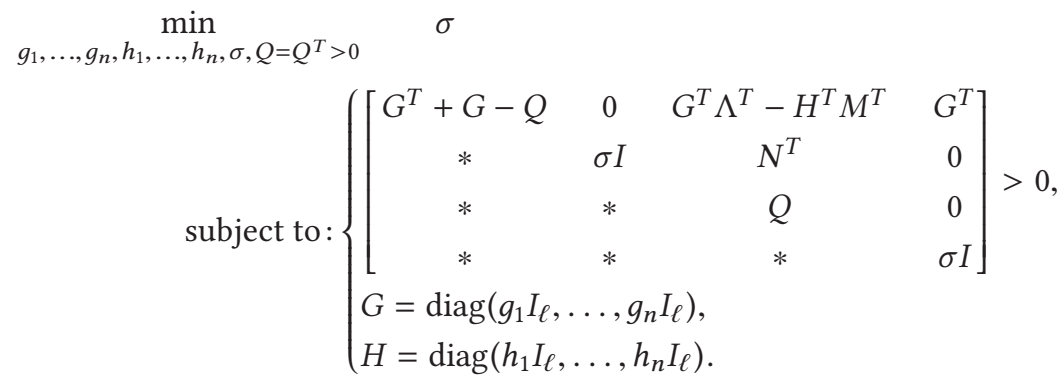

Unlike the CCL-based algorithm, this convex optimization problem searches for both the attenuation factor $\sigma$ and the observer gain $K$ simultaneously. In Section 9, we compare the computation and robustness performance of the two proposed design approaches. We conclude this section with the following theorem.

THEOREM 7.7. Consider the MML observer defined in Equation (7.2). If there exist diagonal matrices $G$ and $H$ and a positive definite matrix $Q=Q^{T}>0$ such that Problem 7.6 is feasible, then the bilinear constraint (7.4) holds. Therefore, Problem 7.6 is a convex relaxation of Problem 7.3, hence Problem 7.1.

\section{NUMERICAL EVALUATION}

As shown by Theorem 4.2, the memory requirement of our observer architecture grows linearly with the number of sensors and system states, a substantial improvement over traditional architectures. In fact, by assuming a number of sensors ranging from 500 to 5,000 with 100 sensors being under attack, previously proposed observer- or filter-based algorithms (Pasqualetti et al. 2013; Mishra et al. 2015) would not be directly implementable, since they would require a bank of $\left(\begin{array}{l}500 \\ 400\end{array}\right)=2.0417 \times 10^{107}$ observers or filters for a system with 500 sensors. Since each observer or filter updates a vector of $n$ elements, this results in $2.0417 \times 10^{107} \times n$ memory units to implement a traditional architecture. Similarly, for a system with 5,000 sensors, previous algorithms require $\left(\begin{array}{l}5,000 \\ 4,900\end{array}\right) \times n=3.1201 \times 10^{211} \times n$ memory units. Our MML observer uses, instead, only $500 \times n$ memory units for a system with 500 sensors or $5,000 \times n$ memory units for a system with 5,000 sensors, which is a substantial decrease in terms of memory requirements.

Therefore, in this section, we focus on the evaluation of the time required to search for the attack-free sensors, and compare the performance of the proposed observer against the solution obtained by (i) our previously proposed IмнотеP-SMT algorithm, which uses data collected over a finite window length; and (ii) the $L_{1} \backslash L_{r}$ algorithm (Fawzi et al. 2014). Since the search space increases exponentially with the number of sensors $p$, we generate a set of random systems (i.e., random matrices $A, B$, and $C$ ) for an increasing number of sensors $p$, assuming that the number of states $n$ is fixed. For each system, we generate a random initial state $x^{(0)}$ and input sequence $u^{(t)}$. 


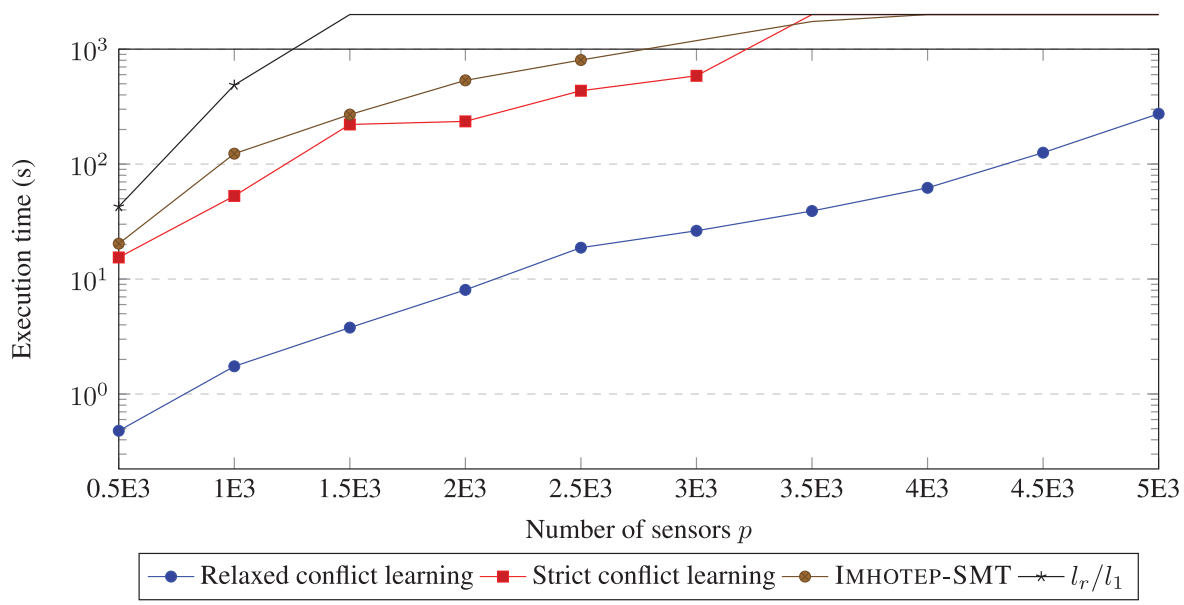

Fig. 5. Worst case execution time for different algorithms.

We run the experiments multiple times, by randomly selecting each time 100 sensors as being under attack. We report the worst case execution time in Figure 5. All the experiments were executed on an Intel Core i7 3.4-GHz processor with 8GB of memory. The proposed observers are implemented in MATLAB, while the pseudo-Boolean SAT solver is implemented using the SAT4J solver (Berre and Parrain 2010).

As shown in Figure 5, the relaxed conflict clause learning algorithm outperforms the other algorithms by at least an order of magnitude. Moreover, as the number of sensors increases, the gap between the relaxed conflict clause learning algorithm and other algorithms increases. The relaxed learning algorithm takes approximately 274 s, in the worst case, for a system with 5,000 sensors, which shows that our approach is indeed suitable to be deployed on large-scale systems. On the other hand, the worst case execution time of the strict learning algorithm is comparable to that of the finite-window-length algorithm. This is mostly due to the fact that, in the worst case, the strict learning algorithm may end up with finding all the needed conflict clauses to reveal the attack-free set, after which no further learning is needed.

\section{CASE STUDY: POWER SYSTEMS}

Power systems are an important example of CPSs for which attacks have been recently documented (Liu et al. 2009). In this article, we consider the IEEE 14-bus power network shown in Figure 6 (left), composed of 5 synchronous generators and 14 buses. The state of each generator includes rotor angle and frequency. The overall system has 35 sensors: 14 sensors measure the real power injections at every bus, 20 sensors measure the real power flows along every branch, and 1 sensor measures the rotor angle of generator 1 . The matrices $A, B$, and $C$ modeling the power network are derived by Pasqualetti et al. (2013). While the IEEE 14-bus can model the power generation and distribution within a small geographical area, our objective is to test the robustness performance of the proposed algorithms for large-scale systems. In fact, a major concern in secure state estimation is the ability of an intruder to use the uncertainty in the model and the noise in sensors to mount its attack (Pajic et al. 2014). To emulate the power generation and distribution over a larger geographical area, we instantiate the IEEE 14-bus system multiple times and connect these instances together, as shown in Figure 6 (right).

Since the scalability of our algorithm is evaluated in detail in Section 8, in this case study we focus on the robustness performance of the proposed observer against process and sensor noise 


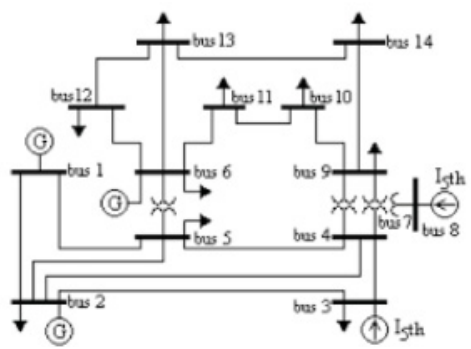

(a)

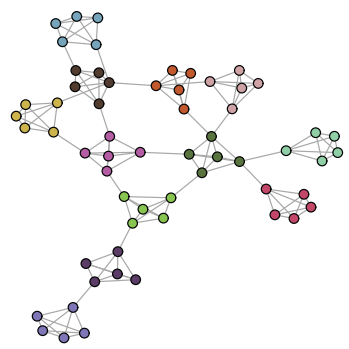

(b)

Fig. 6. (a) The IEEE 14-bus power network and (b) 12 connected instances of the IEEE 14 bus where each set of colored nodes represents the five generators in an IEEE 14 bus.

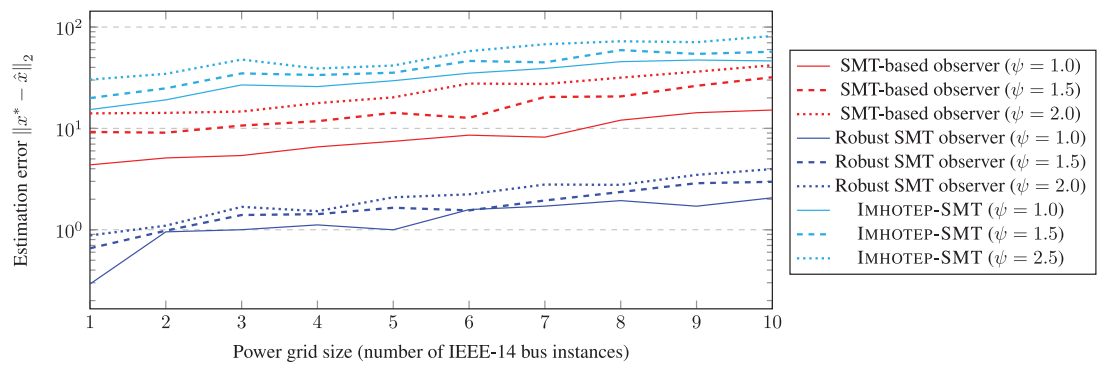

Fig. 7. Norm of estimation error $\left\|x^{(t)}-\hat{x}^{(t)}\right\|_{2}$ in a semi-logarithmic scale (evaluated at the end of simulation time) in the power grid test case for (i) SMT-based observer whose poles are uniformly covering the unit disk, (ii) a robust SMT-based observer designed using the SSM-based algorithm, and (iii) IMHOTEP-SMT, a finitewindow-length algorithm.

as the size of the power grid and the sensor noise magnitude increase. In particular, we compare the estimation estimation error obtained by (i) the proposed SMT-observer architecture with design parameters $k_{1}, \ldots, k_{n}$ set to uniformly cover the unit disk; (ii) the robust SMT-observer, where $k_{1}, \ldots, k_{n}$ are chosen using the SSM-based algorithms in Section 7; and (iii) the previously proposed IмнотеP-SMT solver (Shoukry et al. 2014; Shoukry et al. 2015), which uses sensor data collected over a finite window length.

Similarly to the numerical evaluation in Section 8, we run the experiments multiple times, by randomly selecting one-third of the sensors as being under attack. We report the average estimation error in Figure 7 as a function of the power grid size for three different sensor noise magnitudes. As shown in Figure 7, the proposed SMT-based observers perform better than IMHOTEP-SMT, which is to be expected as the observer averages out the noise over time. Moreover, the robust observer design leads to more than one order of magnitude noise attenuation compared to IMHотEP-SMT. This advantage becomes substantial as the size of the system (hence, the norm bound $\left.\bar{\psi}^{2}=\sum_{i=1}^{p} \bar{\psi}_{i}\right)$ increases.

\section{CONCLUSIONS}

We addressed the problem of designing large-scale cyber-physical systems that are resilient to sensor attacks. We proposed a novel, scalable, MML observer that can isolate the sensors under attack and estimate the state of the underlying dynamics from the remaining sensors. Our architecture has a memory requirement that scales linearly with the system size and adopts an efficient 
SMT-based search algorithm to harness the computational complexity of identifying the set of attacked sensors. Moreover, it leverages two optimization-based algorithms to efficiently select the observer parameters with the goal of minimizing the sensitivity of the estimates with respect to sensor noise. Numerical results show that the proposed observer outperforms other state-of-theart algorithms in terms of memory requirements and computational complexity, and is suitable to be deployed on large-scale systems. When applied to a power grid case study, the MML observer shows a substantial improvement in estimation error in the presence of both model uncertainties and sensor noise.

\section{A ANALYSIS OF THE ATTACK-FREE LUENBERGER OBSERVER}

In this Appendix, we study the convergence of the attack-free Luenberger Observer in the presence of a norm-bounded process and measurement noise. We start with the following intermediate result.

Proposition A.1. Consider the following linear dynamical system:

$$
x^{(t+1)}=A x^{(t)}+u^{(t)}
$$

where $A$ is a stable matrix. Then, there exist constants $0<\bar{\lambda}<1$ and $\kappa \in \mathbb{R}^{+}$such that the function $V\left(x^{(t)}\right)=x^{(t)^{T}} P x^{(t)}$ with $P=P^{T}>0$ satisfies

$$
V\left(x^{(t+1)}\right) \leq \bar{\lambda} V\left(x^{(t)}\right)+\kappa\left\|u^{(t)}\right\|_{2}^{2}
$$

and $A^{T} P A-P=-I$.

Proof. The proof follows a similar argument as the proof of Lemma 1 in Liberzon (2014) and hence is omitted for brevity.

Using the result above, we can study the convergence of the Luenberger observer that uses the attack-free set of sensors. In particular, we can bound the norm of the estimation error as shown in the following result.

Proposition A.2. Let $\Gamma^{\star}$ denote the set of $p-\bar{s}$ sensors which are attack-free for all time $t \in \mathbb{N}$. Let $\hat{x}_{\Gamma^{\star}}^{(t)}$ be the state estimated by the Luenberger observer that uses only the sensors indexed by $\Gamma^{\star}$. The following holds for any sensor $i \in \Gamma^{\star}$ and for all $t \in \mathbb{N}$ :

$$
\left\|Y_{i}^{(t)}-O_{i} \hat{x}_{\Gamma^{\star}}^{(t)}\right\|_{2}^{2} \leq \gamma_{i} \bar{\lambda}^{t}+d_{i}\left(t, \bar{\psi}^{2}, \bar{\mu}^{2}\right),
$$

where

$$
\begin{aligned}
& \left(A-L_{\Gamma} C_{\Gamma}\right)^{T} P_{\Gamma}\left(A-L_{\Gamma} C_{\Gamma}\right)-P_{\Gamma}=-I, \quad d_{i}\left(t, \bar{\psi}^{2}, \bar{\mu}^{2}\right)=\alpha_{i} \sum_{m=0}^{t-1} \bar{\lambda}^{t-m-1}+\beta_{i}, \\
& \bar{\lambda}_{P}=\max _{\substack{\Gamma \subset\{1, \ldots, p\} \\
|\Gamma|=p-\bar{s}}} \lambda_{\max }\left\{P_{\Gamma}\right\}, \quad \underline{\lambda}_{P}=\min _{\substack{\Gamma \subset\{1, \ldots, p\} \\
|\Gamma|=p-\bar{s}}} \lambda_{\min }\left\{P_{\Gamma}\right\}, \quad \gamma_{i}=\frac{2\left\|O_{i}\right\|_{2} \bar{\lambda}_{P}\left\|Y^{(0)}\right\|_{2}^{2}}{\underline{\lambda}_{P} \delta_{2 \bar{s}}^{2}}, \\
& \overline{\bar{\Psi}}=\bar{\mu}+\bar{\psi} \max _{\substack{\Gamma \subset\{1, \ldots, p\},|\Gamma|=p-\bar{s}}} \ln _{\Gamma} \|_{2}, \quad L_{i}=\frac{2\left\|O_{i}\right\|_{2} \kappa \bar{\Psi}^{2}}{\lambda_{P}}, \quad \beta_{i}=2 \bar{\Psi}_{i}^{2}, \quad \bar{\lambda}=1-\frac{1}{2 \bar{\lambda}_{P}}, \\
& \kappa=\max _{\substack{\Gamma \subset\{1, \ldots, p\} \\
|\Gamma|=p-\bar{s}\}}}^{\left\|P_{\Gamma}\left(A-L_{\Gamma} C_{\Gamma}\right)\right\|_{2}^{2}+\left\|P_{\Gamma}\right\|_{2} .},
\end{aligned}
$$


Proof. We start by writing the error dynamics of the Luenberger observer corresponding to $\Gamma^{\star}$ as

$$
e_{x}^{(t+1)}=x^{(t+1)}-\hat{x}_{\Gamma^{\star}}^{(t+1)}=A_{e} e_{x}^{(t)}+\mu^{(t)}+L_{\Gamma^{\star}} \psi_{\Gamma^{\star}}^{(t)},
$$

where $e_{x}^{(t)}=x^{(t)}-\hat{x}_{\Gamma^{\star}}^{(t)}$ and $A_{e}=A-L_{\Gamma^{\star}} C_{\Gamma^{\star}}$. It follows from the $2 \bar{s}$-sparse observability property that the pair $\left(A, C_{\Gamma^{\star}}\right)$ is observable, hence there exists an observer gain $L^{\star}$ such that the error dynamics (A.2) is stable. Therefore, by applying Proposition A.1 we conclude that there exists a function $V\left(e_{x}^{(t)}\right)=e_{x}^{(t)^{T}} P e_{x}^{(t)}$ such that

$$
V\left(e_{x}^{(t+1)}\right) \leq \bar{\lambda} V\left(e_{x}^{(t)}\right)+\kappa\left\|\mu^{(t)}+L_{\Gamma^{\star}} \psi_{\Gamma^{\star}}^{(t)}\right\|_{2}^{2} \leq \bar{\lambda} V\left(e_{x}^{(t)}\right)+\kappa^{\overline{\bar{\Psi}}^{2}}
$$

where the second inequality follows from the definition of $\overline{\bar{\Psi}}$ and the fact that

$$
\left\|\mu^{(t)}+L_{\Gamma^{\star}} \psi_{\Gamma^{\star}}^{(t)}\right\|_{2} \leq\left\|\mu^{(t)}\right\|_{2}+\left\|L_{\Gamma^{\star}}\right\|_{2}\left\|\psi_{\Gamma^{\star}}^{(m)}\right\|_{2} \leq \overline{\bar{\Psi}} .
$$

By rolling forward the dynamics, we can write the function at time $t$ as

$$
V\left(e_{x}^{(t)}\right) \leq \bar{\lambda}^{t} V\left(e_{x}^{(0)}\right)+\kappa \overline{\bar{\Psi}}^{2} \sum_{m=0}^{t-1} \bar{\lambda}^{t-m-1},
$$

from which we conclude that

$$
\left\|e_{x}^{(t)}\right\|_{2}^{2} \leq \frac{\lambda_{\max }\{P\}}{\lambda_{\min }\{P\}} \bar{\lambda}^{t}\left\|e^{(0)}\right\|_{2}^{2}+\frac{\kappa \overline{\bar{\Psi}}^{2}}{\lambda_{\min }\{P\}} \sum_{m=0}^{t-1} \bar{\lambda}^{t-m-1} .
$$

Accordingly, for each sensor $i \in \Gamma^{\star}$ the following bound holds:

$$
\begin{aligned}
\left\|Y_{i}^{(t)}-O_{i} \hat{x}^{(t)}\right\|_{2}^{2} & =\left\|O_{i} e_{x}^{(t)}+\Psi_{i}\right\|_{2}^{2} \stackrel{(a)}{\leq} 2\left\|O_{i} e_{x}^{(t)}\right\|_{2}^{2}+2 \bar{\Psi}_{i}^{2} \leq 2\left\|O_{i}\right\|_{2}\left\|e_{x}^{(t)}\right\|_{2}^{2}+2 \bar{\Psi}_{i}^{2} \\
& \stackrel{(b)}{\leq} \frac{2\left\|O_{i}\right\|_{2} \lambda_{\max }\{P\}}{\lambda_{\min }\{P\}}\left\|e^{(0)}\right\|_{2}^{2} \bar{\lambda}^{t}+\frac{2\left\|O_{i}\right\|_{2} \kappa \bar{\Psi}^{2}}{\lambda_{\min }\{P\}} \sum_{m=0}^{t-1} \bar{\lambda}^{t-m-1}+2 \bar{\Psi}_{i}^{2} \\
& \stackrel{(c)}{\leq} \frac{2\left\|O_{i}\right\|_{2} \lambda_{\max }\{P\}}{\lambda_{\min }\{P\}} \frac{\left\|Y^{(0)}\right\|_{2}^{2}}{\delta_{2 \bar{s}}^{2}} \bar{\lambda}^{t}+\frac{2\left\|O_{i}\right\|_{2} \kappa \overline{\bar{\Psi}}^{2}}{\lambda_{\min }\{P\}} \sum_{m=0}^{t-1} \bar{\lambda}^{t-m-1}+2 \bar{\Psi}_{i}^{2} \\
& =\gamma_{i} \bar{\lambda}^{t}+\alpha_{i} \sum_{m=0}^{t-1} \bar{\lambda}^{t-m-1}+\beta_{i},
\end{aligned}
$$

where (a) follows from the Cauchy-Schwarz inequality along with the definition of $\bar{\Psi}_{i}$; $(b)$ follows from (A.3); and (c) follows from the fact that the Luenberger observer is initialized at zero, hence we obtain

$$
\left\|Y_{\Gamma^{\star}}^{(0)}-O_{\Gamma^{\star}} \hat{x}^{(0)}\right\|_{2}=\left\|Y_{\Gamma^{\star}}^{(0)}\right\|_{2}=\left\|O_{\Gamma^{\star}} x^{(0)}\right\|_{2} \geq \lambda_{\min }\left\{O_{\Gamma^{\star}}^{T} O_{\Gamma^{\star}}\right\}\left\|e_{x}^{(0)}\right\|_{2} \geq \delta_{2 \bar{s}}\left\|e_{x}^{(0)}\right\|_{2},
$$

combined with the fact that $\left\|Y^{(0)}\right\|_{2} \geq\left\|Y_{\Gamma^{\star}}^{(0)}\right\|_{2}$. 


\section{REFERENCES}

D. L. Berre and A. Parrain. 2010. The sat4j library, release 2.2. fournal on Satisfiability, Boolean Modeling and Computation 7 (2010), 59-64.

M. S. Chong, M. Wakaiki, and J. P. Hespanha. 2014. Observability of linear systems under adversarial attacks. In American Control Conference (ACC'15). 2439-2444.

B. De Schutter. 2000. Minimal state-space realization in linear system theory: An overview. Journal of Computational and Applied Mathematics 121, 1-2 (2000), 331-354.

C. E. de Souza and L. Xie. 1992. On the discrete-time bounded real lemma with application in the characterization of static state feedback $H_{\infty}$ controllers. Systems \& Control Letters 18, 1 (1992), 61-71.

L. El Ghaoui, F. Oustry, and M. AitRami. 1997. A cone complementarity linearization algorithm for static output-feedback and related problems. IEEE Transactions on Automatic Control 42, 8 (1997), 1171-1176.

S. Farahmand, G. B. Giannakis, and D. Angelosante. 2011. Doubly robust smoothing of dynamical processes via outlier sparsity constraints. IEEE Transactions on Signal Processing 59, 10 (Oct. 2011), 4529-4543. DOI : http://dx.doi.org/10.1109/ TSP.2011.2161300

H. Fawzi, P. Tabuada, and S. Diggavi. 2014. Secure estimation and control for cyber-physical systems under adversarial attacks. IEEE Transactions on Automatic Control 59, 6 (June 2014), 1454-1467. DOI : http://dx.doi.org/10.1109/TAC.2014. 2303233

R. Langner. 2011. Stuxnet: Dissecting a cyberwarfare weapon. IEEE Security and Privacy Magazine 9, 3 (2011), 49-51.

D. Liberzon. 2014. Finite data-rate feedback stabilization of switched and hybrid linear systems. Automatica 50, 2 (Feb. 2014), 409-420.

Y. Liu, P. Ning, and M. K. Reiter. 2009. False data injection attacks against state estimation in electric power grids. In Proceedings of the 16th ACM conference on Computer and Communications Security (CCS'09). ACM, New York, 21-32.

S. Malik and L. Zhang. 2009. Boolean satisfiability from theoretical hardness to practical success. Communications of the ACM 52, 8 (Aug. 2009), 76-82.

J. Mattingley and S. Boyd. 2010. Real-time convex optimization in signal processing. IEEE Signal Processing Magazine 27, 3 (May 2010), 50-61. DOI : http://dx.doi.org/10.1109/MSP.2010.936020

S. Mishra, Y. Shoukry, N. Karamchandani, S. Diggavi, and P. Tabuada. 2015. Secure state estimation: Optimal guarantees against sensor attacks in the presence of noise. In Proceedings of the IEEE International Symposium on Information Theory (ISIT).

Y. Mo and R. M. Murray. 2015. Multi-dimensional state estimation in adversarial environment. In Proceedings of the 34th Chinese Control Conference (CCC). 4761-4766.

P. Nuzzo, A. Sangiovanni-Vincentelli, D. Bresolin, L. Geretti, and T. Villa. 2015. A platform-based design methodology with contracts and related tools for the design of cyber-physical systems. Proceedings of the IEEE 103, 11 (Nov. 2015), 2104-2132.

M. Pajic, J. Weimer, N. Bezzo, P. Tabuada, O. Sokolsky, Insup Lee, and G. J. Pappas. 2014. Robustness of attackresilient state estimators. In Proceedings of the ACM/IEEE International Conference on Cyber-Physical Systems (ICCPS'14). 163-174.

F. Pasqualetti, F. Dorfler, and F. Bullo. 2013. Attack detection and identification in cyber-physical systems. IEEE Transactions on Automatic Control 58, 11 (Nov. 2013), 2715-2729. DOI : http://dx.doi.org/10.1109/TAC.2013.2266831

Y. Shoukry, M. Chong, M. Wakaiki, P. Nuzzo, A. L. Sangiovanni-Vincentelli, S. A. Seshia, J. P. Hespanha, and P. Tabuada. 2016. SMT-Based observer design for cyber-physical systems under sensor attacks. In Proceedings of the International Conference on Cyber-Physical Systems. IEEE, 1-10.

Y. Shoukry, P. D. Martin, P. Tabuada, and M. B. Srivastava. 2013. Non-invasive spoofing attacks for anti-lock braking systems. In Proceedings of the Workshop on Cryptographic Hardware and Embedded Systems (CHES'13), G. Bertoni and J.-S. Coron (Eds.), Lecture Notes in Computer Science, Vol. 8086. International Association for Cryptologic Research, $55-72$.

Y. Shoukry, P. Nuzzo, A. Puggelli, A. L. Sangiovanni-Vincentelli, S. A. Seshia, and P. Tabuada. 2014. Secure state estimation under sensor attacks: A satisfiability modulo theory approach. (2014). arXiv:math.OC/1412.4324 [online], http://arxiv. org/abs/1412.4324.

Y. Shoukry, A. Puggelli, P. Nuzzo, A. L. Sangiovanni-Vincentelli, S. A. Seshia, and P. Tabuada. 2015. Sound and complete state estimation for linear dynamical systems under sensor attack using satisfiability modulo theory solving. In Proceedings of the IEEE American Control Conference. 3818-3823.

Y. Shoukry and P. Tabuada. 2016. Event-triggered state observers for sparse sensor noise/attacks. IEEE Transactions on Automatic Control 61, 8 (2016), 2079-2091. http://ieeexplore.ieee.org/xpl/articleDetails.jsp?arnumber=7308014

Roy S. Smith. 2011. A decoupled feedback structure for covertly appropriating networked control systems. IFAC Proceedings Volumes 44, 1 (2011), 90-95. 
A. Teixeira, Kin Cheong Sou, H. Sandberg, and K. H. Johansson. 2015. Secure control systems: A quantitative risk management approach. IEEE Control Systems Magazine 35, 1 (Feb. 2015), 24-45. DOI : http://dx.doi.org/10.1109/MCS.2014. 2364709

Sze Zheng Yong, Minghui Zhu, and Emilio Frazzoli. 2015. Resilient state estimation against switching attacks on stochastic cyber-physical systems. In Proceedings of the 54th IEEE Conference on Decision and Control (CDC'15). IEEE, 5162-5169.

Quanyan Zhu and Tamer Başar. 2011. Robust and resilient control design for cyber-physical systems with an application to power systems. In Proceedings of the 50th IEEE Conference on Decision and Control and European Control Conference. IEEE, 4066-4071.

Received July 2016; revised January 2017; accepted April 2017 